\title{
Integrable systems and the boundary dynamics of higher spin gravity on $\mathrm{AdS}_{3}$
}

\author{
Emilio Ojeda ${ }^{a, b}$ and Alfredo Pérez ${ }^{a}$ \\ ${ }^{a}$ Centro de Estudios Científicos (CECs), \\ Avenida Arturo Prat 514, Valdivia, Chile \\ ${ }^{b}$ Departamento de Física, Universidad de Concepción, \\ Casilla 160-C, Concepción, Chile \\ E-mail: ojeda@cecs.cl, aperez@cecs.cl
}

ABSTRACT: We introduce a new set of boundary conditions for three-dimensional higher spin gravity with gauge group $\mathrm{SL}(3, \mathbb{R}) \times \mathrm{SL}(3, \mathbb{R})$, where its dynamics at the boundary is described by the members of the modified Boussinesq integrable hierarchy. In the asymptotic region the gauge fields are written in the diagonal gauge, where the excitations go along the generators of the Cartan subalgebra of $\operatorname{sl}(3, \mathbb{R}) \oplus \operatorname{sl}(3, \mathbb{R})$. We show that the entire integrable structure of the modified Boussinesq hierarchy, i.e., the phase space, the Poisson brackets and the infinite number of commuting conserved charges, are obtained from the asymptotic structure of the higher spin theory. Furthermore, its known relation with the Boussinesq hierarchy is inherited from our analysis once the asymptotic conditions are re-expressed in the highest weight gauge. Hence, the Miura map is recovered from a purely geometric construction in the bulk. Black holes that fit within our boundary conditions, the Hamiltonian reduction at the boundary, and the generalization to higher spin gravity with gauge group $\mathrm{SL}(N, \mathbb{R}) \times \mathrm{SL}(N, \mathbb{R})$ are also discussed.

KEYwords: Higher Spin Gravity, Gauge-gravity correspondence, Black Holes

ArXiv EPrint: 2009.07829 


\section{Contents}

1 Introduction 1

2 Review of the modified Boussinesq hierarchy 3

3 Modified Boussinesq hierarchy from spin-3 gravity on AdS $_{3} \quad 6$

3.1 Chern-Simons formulation of spin-3 gravity on $\mathrm{AdS}_{3} \quad 6$

$\begin{array}{lll}3.2 & \text { Asymptotic behavior of the fields. Diagonal gauge } & 7\end{array}$

3.3 Boundary conditions for spin-3 gravity and the modified Boussinesq hierarchy 7

3.4 Asymptotic symmetries and conserved charges 8

3.5 Highest weight gauge, Miura map and the Boussinesq hierarchy 10

4 Black holes $\quad 12$

5 Hamiltonian reduction and boundary dynamics $\quad 14$

6 Some extensions: Generalized Gibbs ensemble, spin-N gravity and mod$\begin{array}{lr}\text { ified Gelfand-Dickey hierarchies } & \mathbf{1 6}\end{array}$

6.1 Generalized Gibbs Ensemble 16

6.2 Higher spin gravity with gauge group $\mathrm{SL}(N, \mathbb{R}) \times \mathrm{SL}(N, \mathbb{R})$ and modified $\begin{array}{ll}\text { Gelfand-Dickey hierarchies } & 17\end{array}$

$\begin{array}{lll}7 & \text { Final remarks } & 18\end{array}$

A Second Hamiltonian structure of the modified Boussinesq hierarchy $\quad 19$

B Gelfand-Dickey polynomials and Hamiltonians of the modified Boussi$\begin{array}{ll}\text { nesq hierarchy } & 19\end{array}$

$\begin{array}{lr}\text { C Boussinesq hierarchy } & 20\end{array}$

D Fundamental representation of the principal embedding of $\operatorname{sl}(2, \mathbb{R})$ within $\operatorname{sl}(N, \mathbb{R}) \quad 22$

E Wess-Zumino term $\quad 22$

\section{Introduction}

The asymptotic structure of spacetime plays a fundamental role in the description of General Relativity in three dimensions. This theory does not possess local propagating degrees of freedom, and consequently, its dynamics is completely dictated by the choice of boundary conditions. In the case of a negative cosmological constant, it is standard practice the 
use of the ones of Brown and Henneaux [1], whose asymptotic symmetries are spanned by two copies of the Virasoro algebra with central charge $c=3 l / 2 G$. However, this choice is not unique. There are other possible sensible boundary conditions that can be consistently used, with different physical consequences [2-9]. In particular, the ones proposed in ref. [6] relate the dynamics of the gravitational field with the two-dimensional Korteweg-de Vries (KdV) integrable hierarchy. Therefore, the asymptotic symmetries are spanned by the infinite set of commuting KdV charges, which are composite in terms of the Virasoro generators. This framework allows for example to study Generalized Gibbs Ensembles of two-dimensional conformal field theories in terms of a gravitational dual [6, 10], as well as constructing black hole solutions carrying non-trivial KdV charges [11].

Different extensions of this relation between a three-dimensional gravitational theory in the bulk with specific boundary conditions, and an integrable system at the boundary, were also studied in ref. [12] for General Relativity with a vanishing cosmological constant, in ref. [13] for gravity on AdS coupled to two U(1) Chern-Simons fields, and in ref. [8] for pure gravity on AdS in connection with the Gardner hierarchy.

It is then natural to explore the possibility of generalize these results to the case of General Relativity coupled to higher spin fields [14-16]. Indeed, in ref. [17] a connection between spin-3 gravity and a "Boussinesq equation in the light-cone" was pointed out. In refs. $[18,19]$, some particular boundary conditions for higher spin gravity with gauge group $\operatorname{SL}(N, \mathbb{R}) \times \mathrm{SL}(N, \mathbb{R})$ were associated to a generalized $\mathrm{KdV}$ hierarchy, and some particular cases, including the Boussinesq equation, were explicitly worked out. On the other hand, in ref. [6] a very precise link with the Boussinesq hierarchy was described for spin-3 gravity, as well as for its extension including fields with arbitrary higher spins. The analysis was based on boundary conditions defined in the highest weight gauge with a particular choice of chemical potentials, generalizing the results obtained for the KdV hierarchy in pure gravity.

The Boussinesq equation was first introduced by Joseph Boussinesq in 1872 in the context of the study of the propagation of one-dimensional long waves in shallow water, moving in both directions [20]. Long after, in 1974, it was realized that the equation was integrable, and that belongs to a hierarchy of differential equations [21]. Most of the important properties of the Boussinesq equation, including its infinite set of commuting conserved charges, can be easily derived when a potential equation, called "modified Boussinesq" (mBoussinesq), is introduced (see e.g [22]). Both equations are then related by an appropriate generalization of the Miura transformation [23-25].

One of the purposes of our work is to show that the asymptotic dynamics of spin-3 gravity on $\mathrm{AdS}_{3}$ endowed with a special class of boundary conditions, is precisely described by the members of the mBoussinesq hierarchy. In this framework, the gauge fields are defined in the "diagonal gauge," where the excitations go along the generators of the Cartan subalgebra of $s l(3, \mathbb{R}) \oplus \operatorname{sl}(3, \mathbb{R})[26]$. The link with the integrable system is then obtained by choosing the chemical potentials as precise functionals of the dynamical fields, in a way consistent with the action principle. Hence, the entire integrable structure of the mBoussinesq hierarchy, i.e., the phase space, the fundamental Poisson brackets given by two independent $\hat{u}(1)$ current algebras, and the infinite set of Hamiltonians in involution, are 
obtained from the asymptotic structure of the higher spin theory in the bulk. Furthermore, the relation with the Boussinesq hierarchy previously found in ref. [6] is inherited from our analysis once the asymptotic conditions are re-expressed in the highest weight gauge along the lines of ref. [26]. Thus, the Miura map is recovered from a purely geometric construction in the higher spin theory. Black hole solutions that fit within our boundary conditions, the Hamiltonian reduction at the boundary, and the generalization to higher spin gravity with gauge group $\mathrm{SL}(N, \mathbb{R}) \times \mathrm{SL}(N, \mathbb{R})$ are also discussed.

The plan of the paper is the following. In the next section we briefly review the biHamiltonian formulation of the mBoussinesq hierchy and its relation with the Boussinesq one. In section 3 we propose a new set of boundary conditions for higher spin gravity on $\mathrm{AdS}_{3}$ with gauge group $\mathrm{SL}(3, \mathbb{R}) \times \mathrm{SL}(3, \mathbb{R})$, such that the asymptotic dynamics is precisely described by a particular member of the mBoussinesq hierarchy. All the relevant properties of the integrable system, including its infinite number of commuting conserved charges, are derived from the theory in the bulk. Section 4 is devoted to the study of black hole configurations that fit within our boundary conditions. It is shown that they are generically described by static solutions of the corresponding member of the mBoussinesq hierarchy. The regularity conditions that come from requiring trivial holonomies around the thermal cycle, as well as their thermodynamic properties are also analyzed. In section 5 we perform the Hamiltonian reduction of the Chern-Simons action describing spin-3 gravity with the boundary conditions associated to the mBoussinesq hierarchy. It is shown that the boundary dynamics, and in particular the non-trivial interacting terms in the reduced action are completely determined by the Hamiltonians of the hierarchy. In section 6 we discuss two possible extensions of our previous results. We first describe a natural generalization of the boundary conditions that allows to study Generalized Gibbs Ensembles by considering a general Hamiltonian consisting of a linear combination of all the charges in involution of the hierarchy. We also extend our results to the case of three-dimensional higher spin gravity with gauge group $\mathrm{SL}(N, \mathbb{R}) \times \mathrm{SL}(N, \mathbb{R})$, where the associated hierarchy correspond to the $N$-th "Gelfand-Dickey hierarchy." Some final remarks and further possible extensions are briefly addressed in section 7. Five appendices are included. In appendix A the operator that defines the second Poisson structure of the mBoussinesq hierarchy is exhibited. In appendix B the first Hamiltonians and Gelfand-Dickey polynomials of the mBoussinesq hierarchy are displayed. Appendix $\mathrm{C}$ is devoted to a brief review of the Boussinesq hierarchy. In appendix $\mathrm{D}$, the fundamental matrix representation of $\mathrm{SL}(2, \mathbb{R})$ within $\mathrm{SL}(N, \mathbb{R})$, the principal embedding is presented. Finally, in appendix E it is shown that for our boundary conditions the Wess-Zumino term in the Hamiltonian reduction in section 5 vanishes.

\section{Review of the modified Boussinesq hierarchy}

The first member of the mBoussinesq hierarchy is given by the following set of differential equations

$$
\begin{aligned}
& \dot{\mathcal{J}}=\lambda_{1} \mathcal{J}^{\prime}-\lambda_{2}\left(2(\mathcal{J U})^{\prime}+\mathcal{U}^{\prime \prime}\right), \\
& \dot{\mathcal{U}}=\lambda_{1} \mathcal{U}^{\prime}+\lambda_{2}\left(\mathcal{U}^{2 \prime}-\mathcal{J}^{2 \prime}+\mathcal{J}^{\prime \prime}\right)
\end{aligned}
$$


Here dots and primes denote derivatives with respect to the time $t$ and the angle $\phi$ respectively, and $\lambda_{1}, \lambda_{2}$ are arbitrary constants associated to the two different flows of the hierarchy [24]. The case with $\lambda_{1}=0$ and $\lambda_{2}=1$ is known as the mBoussinesq equation, while the case with $\lambda_{1}=1$ and $\lambda_{2}=0$ describes two independent chiral fields.

The dynamics of the above equations may be described using the Hamiltonian formalism. If the Poisson brackets of two arbitrary functional $F$ and $G$ is given by

$$
\{F, G\}=\frac{4 \pi}{\hat{\kappa}} \int d \phi\left(\frac{\delta F}{\delta \mathcal{J}} \partial_{\phi} \frac{\delta G}{\delta \mathcal{J}}+\frac{\delta F}{\delta \mathcal{U}} \partial_{\phi} \frac{\delta G}{\delta \mathcal{U}}\right)
$$

together with the Hamiltonian

$$
H_{(1)}=\frac{\hat{\kappa}}{4 \pi} \int d \phi\left\{\frac{\lambda_{1}}{2}\left(\mathcal{J}^{2}+\mathcal{U}^{2}\right)+\lambda_{2}\left(\frac{1}{3} \mathcal{U}^{3}-\mathcal{J}^{2} \mathcal{U}-\mathcal{J} \mathcal{U}^{\prime}\right)\right\}
$$

then eq. (2.1) can be rewritten as

$$
\dot{\mathcal{J}}=\left\{\mathcal{J}, H_{(1)}\right\}, \quad \dot{\mathcal{U}}=\left\{\mathcal{U}, H_{(1)}\right\} .
$$

Note that the arbitrary constant $\hat{\kappa}$ does not appear in the differential equations (2.1), however it is useful to introduce it in (2.2) and (2.3) for later convenience.

Alternatively, if we define the operator

$$
\mathcal{D}:=\frac{4 \pi}{\hat{\kappa}}\left(\begin{array}{cc}
\partial_{\phi} & 0 \\
0 & \partial_{\phi}
\end{array}\right),
$$

the equations in (2.1) can be re-written in vector form as follows

$$
\left(\begin{array}{c}
\dot{\mathcal{J}} \\
\dot{\mathcal{U}}
\end{array}\right)=\mathcal{D}\left(\begin{array}{c}
\frac{\delta H_{(1)}}{\delta \mathcal{J}} \\
\frac{\delta H_{(1)}}{\delta \mathcal{U}}
\end{array}\right)
$$

The operator $\mathcal{D}$ in (2.4) defines the symplectic structure in eq. (2.2).

It is worth to emphasize that one of the key points in the relation of this integrable system with higher spin gravity comes from the fact that, according to eq. (2.2), the fundamental Poisson brackets are described by two independent $\hat{u}(1)$ current algebras

$$
\begin{aligned}
& \left\{\mathcal{J}(\phi), \mathcal{J}\left(\phi^{\prime}\right)\right\}=\frac{4 \pi}{\hat{\kappa}} \partial_{\phi} \delta\left(\phi-\phi^{\prime}\right), \\
& \left\{\mathcal{U}(\phi), \mathcal{U}\left(\phi^{\prime}\right)\right\}=\frac{4 \pi}{\hat{\kappa}} \partial_{\phi} \delta\left(\phi-\phi^{\prime}\right) .
\end{aligned}
$$

As we will show below, once appropriate boundary conditions are imposed, this Poisson bracket algebra is obtained from the Dirac brackets in the higher spin theory.

The integrability of (2.1) and the existence of a hierarchy of equations, rely on the fact that this system is actually bi-Hamiltonian. Indeed, there exists an alternative symplectic structure characterized by the following non-local operator

$$
\mathcal{D}_{(2)}=\mathcal{D} M^{\dagger} \mathcal{O} M \mathcal{D}
$$


Here,

$$
M=\left(\begin{array}{cc}
\mathcal{J}+\partial_{\phi} & \mathcal{U} \\
-2 \mathcal{J U}-\frac{1}{2} \mathcal{U} \partial_{\phi}-\frac{3}{2} \mathcal{U}^{\prime} & \mathcal{U}^{2}-\mathcal{J}^{2}-\frac{1}{2} \mathcal{J}^{\prime}-\frac{3}{2} \mathcal{J} \partial_{\phi}-\frac{1}{2} \partial_{\phi}^{2}
\end{array}\right),
$$

and $M^{\dagger}$ is the formal adjoint of $M$ (see e.g. [27]). The operator $\mathcal{O}$ that contains the non-local terms is given by

$$
\mathcal{O}=\frac{2 \hat{\kappa}}{\pi}\left(\begin{array}{cc}
0 & \partial_{\phi}^{-1} \\
\partial_{\phi}^{-1} & 0
\end{array}\right)
$$

Consequently, the Poisson bracket of two arbitrary functionals $F$ and $G$ associated with the operator $\mathcal{D}_{(2)}$ is

$$
\{F, G\}_{2}=\int d \phi\left(\begin{array}{ll}
\frac{\delta F}{\delta \mathcal{J}} & \frac{\delta F}{\delta \mathcal{U}}
\end{array}\right) \mathcal{D}_{(2)}\left(\begin{array}{c}
\frac{\delta G}{\delta \mathcal{J}} \\
\frac{\delta G}{\delta \mathcal{U}}
\end{array}\right) .
$$

The explicit components of $\mathcal{D}_{(2)}$ are exhibited in appendix A.

Equations (2.1) can then be recovered using the Poisson bracket (2.8), together with the Hamiltonian ${ }^{1}$

$$
H_{(0)}=\frac{\hat{\kappa}}{4 \pi} \int d \phi\left(\lambda_{1} \mathcal{J}+\lambda_{2} \mathcal{U}\right) .
$$

This system possess an infinite number of conserved charges in involution that can be constructed from the following recursion relation

$$
R_{(n+1)}=\mathcal{D}^{-1} \mathcal{D}_{(2)} R_{(n)},
$$

where

$$
R_{(n)}=\left(\begin{array}{c}
\frac{\delta H_{(n)}}{\delta \mathcal{J}} \\
\frac{\delta H_{(n)}}{\delta \mathcal{U}}
\end{array}\right)
$$

are the Gelfand-Dickey polynomials associated to the hierarchy. The conserved quantities $H_{(n)}$, with $n$ being a nonnegative integer, are generically decomposed into two flows proportional to the constants $\lambda_{1}$ and $\lambda_{2}$ respectively

$$
H_{(n)}=\sum_{I=1}^{2} \lambda_{I} H_{(n)}^{I}
$$

Then one can prove that the $H_{(k)}^{I}$ are in involution with both Poisson brackets, i.e.,

$$
\left\{H_{(n)}^{I}, H_{(m)}^{J}\right\}=\left\{H_{(n)}^{I}, H_{(m)}^{J}\right\}_{2}=0 .
$$

Furthermore, if we one uses the conserved quantities $H_{(k)}^{I}$ as new Hamiltonians, it is then possible to define a hierarchy of integrable equations labelled by the nonnegative integer $k$ of the form

$$
\begin{aligned}
& \dot{\mathcal{J}}=\left\{\mathcal{J}, H_{(k)}\right\}=\left\{\mathcal{J}, H_{(k-1)}\right\}_{2}, \\
& \dot{\mathcal{U}}=\left\{\mathcal{U}, H_{(k)}\right\}=\left\{\mathcal{U}, H_{(k-1)}\right\}_{2} .
\end{aligned}
$$

\footnotetext{
${ }^{1}$ The coefficients $\lambda_{1}$ and $\lambda_{2}$ in eq. (2.1) are determined by the integration constants obtained by the action of $\mathcal{D}_{(2)}$. In the case of higher members of the hierarchy, the subsequent integration constants may be consistently set to zero as they contribute nothing new (see e.g [24]).
} 
The equations associated to each flow, labelled by the index $I=1,2$, have different scaling properties. Under a Lifshitz scaling transformation with dynamical exponent $z$

$$
t \rightarrow \varepsilon^{z} t, \quad \phi \rightarrow \varepsilon \phi, \quad \mathcal{J} \rightarrow \varepsilon^{-1} \mathcal{J}, \quad \mathcal{U} \rightarrow \varepsilon^{-1} \mathcal{U},
$$

the flow with $I=1$ is invariant for $z=3 k-2$, while the flow with $I=2$ is invariant for $z=3 k-1$.

As explained in the introduction, the mBoussinesq equation is a "potential equation" for the Boussinesq one. Indeed, if $\mathcal{U}$ and $\mathcal{J}$ obey the mBoussinesq equation, then the fields $\mathcal{L}$ and $\mathcal{W}$ defined by the Miura transformation as

$$
\begin{aligned}
\mathcal{L} & =\frac{1}{2} \mathcal{J}^{2}+\frac{1}{2} \mathcal{U}^{2}+\mathcal{J}^{\prime} \\
\mathcal{W} & =\frac{1}{3} \mathcal{U}^{3}-\mathcal{J}^{2} \mathcal{U}-\frac{1}{2} \mathcal{U} \mathcal{J}^{\prime}-\frac{3}{2} \mathcal{J} \mathcal{U}^{\prime}-\frac{1}{2} \mathcal{U}^{\prime \prime}
\end{aligned}
$$

obey the Boussinesq equation given by

$$
\begin{aligned}
\dot{\mathcal{L}} & =2 \mathcal{W}^{\prime}, \\
\dot{\mathcal{W}} & =2 \mathcal{L}^{2 \prime}-\frac{1}{2} \mathcal{L}^{\prime \prime \prime} .
\end{aligned}
$$

The entire Boussinesq hierarchy, including the infinite set of charges in involution, is obtained from the mBoussinesq one by using the Miura transformation (see appendix $\mathrm{C}$ for more details on the Boussinesq hierarchy). It is worth noting that the Miura transformation (2.13) coincides the twisted Sugawara construction of the stress tensor and a spin-3 current in terms of two independent $\mathrm{U}(1)$ currents in a two-dimensional CFT. Hence, using the Poisson brackets (2.5) and the Miura map (2.13), one can show that the (first) Poisson brackets for $\mathcal{L}$ and $\mathcal{W}$ are precisely given by the classical $W_{3}$ algebra.

\section{Modified Boussinesq hierarchy from spin-3 gravity on $\mathrm{AdS}_{3}$}

\subsection{Chern-Simons formulation of spin-3 gravity on $\mathrm{AdS}_{3}$}

Higher spin gravity in 3D has the very special property that, in contrast with their higher dimensional counterparts [28-30], its spectrum can be consistently truncated to a finite number of higher spin fields $[14,15,31,32]$. One of the simplest cases corresponds to a spin-two field non-minimally coupled to a spin-three field, that may be described by a Chern-Simons action for the gauge group SL $(3, \mathbb{R}) \times \mathrm{SL}(3, \mathbb{R})$,

$$
I=I_{C S}\left[A^{+}\right]-I_{C S}\left[A^{-}\right]
$$

where

$$
I_{C S}[A]=\frac{\kappa_{3}}{4 \pi} \int_{\mathcal{M}} \operatorname{tr}\left(A d A+\frac{2}{3} A^{3}\right) .
$$

Here, the level is given by $\kappa_{3}=\kappa / 4=l / 16 G$, where $l$ and $G$ correspond to the AdS radius and the three-dimensional Newton constant respectively, and the trace is in the fundamental representation of the $s l(3, \mathbb{R})$ algebra in the principal embedding (see appendix $\mathrm{D})$. The field equations are then given by the vanishing of the field strength

$$
F^{ \pm}=d A^{ \pm}+A^{ \pm 2}=0 .
$$


The metric and the spin-three field are reconstructed in terms of a generalized dreibein $e:=\frac{l}{2}\left(A^{+}-A^{-}\right)$according to

$$
g_{\mu \nu}=\frac{1}{2} \operatorname{tr}\left(e_{\mu} e_{\nu}\right), \quad \varphi_{\mu \nu \rho}=\frac{1}{3 !} \operatorname{tr}\left(e_{(\mu} e_{\nu} e_{\rho)}\right) .
$$

\subsection{Asymptotic behavior of the fields. Diagonal gauge}

Following refs. [31-33], it is convenient to perform the analysis of the asymptotic symmetries of spin-three gravity in terms of an auxiliary connection depending only on $t$ and $\phi$. For simplicity, and without loss of generality, hereafter we will consider only the "plus copy," and hence the superscript "+" will be omitted. The gauge field $A$ is then written as

$$
A=b^{-1}(d+a) b
$$

where $a=a_{t} d t+a_{\phi} d \phi$ is the auxiliary connection, and $b=b(r)$ is a gauge group element which captures the whole the radial dependence of the gauge connection. The asymptotic analysis will be insensitive to the precise form of $b(r)$.

We will consider asymptotic conditions in the "diagonal gauge," i.e., where all the permissible excitations in the auxiliary connection go along the generators of the Cartan subalgebra of $\operatorname{sl}(3, \mathbb{R})[26]$. Then, it takes the form

$$
a=(\mathcal{J} d \phi+\zeta d t) L_{0}+\frac{\sqrt{3}}{2}(\mathcal{U} d \phi+\zeta \mathcal{U} d t) W_{0} .
$$

The fields $\mathcal{J}$ and $\mathcal{U}$ belong to the spatial components of the auxiliary connection, and hence they are identified as the dynamical fields. On the other hand, $\zeta$ and $\zeta_{\mathcal{U}}$ are defined along the temporal components, and therefore they correspond to the boundary values of the Lagrange multipliers. In ref. [26] the same asymptotic form for the auxiliary connection was used, with the replacement $\mathcal{J}_{(3)} \rightarrow \frac{\sqrt{3}}{2} \mathcal{U}$ and $\zeta_{(3)} \rightarrow \frac{\sqrt{3}}{2} \zeta_{\mathcal{U}}$. However, the boundary conditions will be different. In [26] it was assumed that $\zeta$ and $\zeta_{(3)}$ are kept fixed at the boundary, while here, as we will show in the next subsection, they will acquire a precise functional dependence on the dynamical fields $\mathcal{J}, \mathcal{U}$ and their spatial derivatives.

\subsection{Boundary conditions for spin-3 gravity and the modified Boussinesq hier- archy}

A fundamental requirement in the study of the asymptotic structure of spacetime is that the boundary conditions must be compatible with the action principle. In the canonical formalism one has to add an appropriate boundary term $B_{\infty}$ to the canonical action in order to guarantee that the action principle attains an extremum [34]

$$
I_{\text {can }}[A]=-\frac{\kappa}{16 \pi} \int d t d^{2} x \epsilon^{i j}\left\langle A_{i} \dot{A}_{j}-A_{t} F_{i j}\right\rangle+B_{\infty} .
$$

Following [26], for the action (3.6) and the asymptotic conditions (3.4), (3.5), the variation of the boundary term is given by

$$
\delta B_{\infty}=-\frac{\kappa}{4 \pi} \int d t d \phi\left(\zeta \delta \mathcal{J}+\zeta_{\mathcal{U}} \delta \mathcal{U}\right)
$$


In the absence of ingoing or outgoing radiation, as is the case in three-dimensional higher spin gravity, the boundary term $B_{\infty}$ has to be integrable in a functional sense, i.e., one must be able to "take the delta outside" in (3.7). The precise form in which $\zeta$ and $\zeta_{\mathcal{U}}$ are fixed at the boundary is what defines the boundary conditions. Thus, following [6], in order to make contact with the Boussinesq hierarchy in a way consistent with the action principle, we choose the Lagrange multipliers as

$$
\zeta=\frac{4 \pi}{\kappa} \frac{\delta H_{(k)}}{\delta \mathcal{J}}, \quad \zeta_{\mathcal{U}}=\frac{4 \pi}{\kappa} \frac{\delta H_{(k)}}{\delta \mathcal{U}},
$$

where $H_{(k)}$ is the Hamiltonian associated to the $k$-th element of the mBoussinesq hierarchy. With this choice, the boundary term can be readily integrated, and yields

$$
B_{\infty}=-\int d t H_{(k)} .
$$

Thus, the Hamiltonian of the gravitational theory in the reduced phase space precisely matches the one of the integrable system.

On the other hand, the field equations in the higher spin theory given by the vanishing of the field strength become

$$
\dot{\mathcal{J}}=\zeta^{\prime}, \quad \dot{\mathcal{U}}=\zeta_{\mathcal{U}}^{\prime},
$$

which, by virtue of (3.8), precisely coincide with the differential equations associated to the $k$-th element of the mBoussinesq hierarchy in eq. (2.12), provided the constant $\hat{\kappa}$ in eqs. (2.2) and (2.3) is assumed to depend on the cosmological and Newton constants according to $\hat{\kappa}=\kappa$. Then,

$$
\left(\begin{array}{c}
\dot{\mathcal{J}} \\
\dot{\mathcal{U}}
\end{array}\right)=\mathcal{D}\left(\begin{array}{c}
\frac{\delta H_{(k)}}{\delta \mathcal{J}} \\
\frac{\delta H_{(k)}}{\delta \mathcal{U}}
\end{array}\right)=\left(\begin{array}{l}
\left\{\mathcal{J}, H_{(k)}\right\} \\
\left\{\mathcal{U}, H_{(k)}\right\}
\end{array}\right) .
$$

\subsection{Asymptotic symmetries and conserved charges}

The asymptotic symmetries are determined by set of gauge transformations that preserve the asymptotic form of the gauge connection, with non-vanishing associated charges. The form of the auxiliary connection in eq. (3.5) is preserved by gauge transformations $\delta a=$ $d \lambda+[a, \lambda]$, with parameter

$$
\lambda=\eta L_{0}+\frac{\sqrt{3}}{2} \eta_{\mathcal{U}} W_{0}
$$

There could be some additional terms in the non-diagonal components of the gauge parameter $\lambda$, but they are pure gauge in the sense that there are no generators associated to them, so they can be consistently set to zero.

The preservation of the angular components of the auxiliary connection gives the transformation law of the dynamical fields

$$
\delta \mathcal{J}=\eta^{\prime}, \quad \delta \mathcal{U}=\eta_{\mathcal{U}}^{\prime},
$$

while that the preservation of the temporal components provides the transformation law of the Lagrange multipliers

$$
\delta \zeta=\dot{\eta}, \quad \delta \zeta_{\mathcal{U}}=\dot{\eta}_{\mathcal{U}}
$$


The variation of the conserved charges can be computed using the Regge-Teitelboim method [34], and they are given by the following surface integral

$$
\delta Q\left[\eta, \eta_{\mathcal{U}}\right]=\frac{\kappa}{4 \pi} \int d \phi\left(\eta \delta \mathcal{J}+\eta_{\mathcal{U}} \delta \mathcal{U}\right) .
$$

The Dirac brackets of the dynamical fields $\mathcal{J}$ and $\mathcal{U}$ induced by the asymptotic conditions may be obtained from the relation $\delta_{Y} Q[X]=\{Q[X], Q[Y]\}$, and is given by two independent $\hat{u}(1)$ current algebras

$$
\begin{aligned}
\left\{\mathcal{J}(\phi), \mathcal{J}\left(\phi^{\prime}\right)\right\}^{\star} & =\frac{4 \pi}{\kappa} \partial_{\phi} \delta\left(\phi-\phi^{\prime}\right), \\
\left\{\mathcal{U}(\phi), \mathcal{U}\left(\phi^{\prime}\right)\right\}^{\star} & =\frac{4 \pi}{\kappa} \partial_{\phi} \delta\left(\phi-\phi^{\prime}\right),
\end{aligned}
$$

expression that coincides with the first Poisson bracket of the mBoussinesq hierarchy given by eq. (2.5). Furthermore, the infinite set of commuting charges of the hierarchy is obtained from the surface integral (3.14) as follows: if we take into account that due to eq. (3.8) the Lagrange multipliers are field dependent, then the consistency with their transformation law (3.13) implies the following differential equation that must be obeyed by $\eta$ and $\eta_{\mathcal{U}}$

$$
\left(\begin{array}{c}
\dot{\eta}(t, \theta) \\
\dot{\eta}_{\mathcal{U}}(t, \theta)
\end{array}\right)=\int d \phi\left(\begin{array}{cc}
\frac{\delta^{2} H_{(k)}}{\delta \mathcal{J}(t, \theta) \delta \mathcal{J}(t, \phi)} & \frac{\delta^{2} H_{(k)}}{\delta \mathcal{U}(t, \theta) \delta \mathcal{J}(t, \phi)} \\
\frac{\delta^{2} H_{(k)}}{\delta \mathcal{J}(t, \theta) \delta \mathcal{U}(t, \phi)} & \frac{\delta^{2} H_{(k)}}{\delta \mathcal{U}(t, \theta) \delta \mathcal{U}(t, \phi)}
\end{array}\right) \mathcal{D}\left(\begin{array}{c}
\eta \\
\eta_{\mathcal{U}}
\end{array}\right) .
$$

By virtue of the integrability of the system, the most general solution of this equation, under the assumption that $\eta$ and $\eta_{\mathcal{U}}$ depend locally on $\mathcal{J}, \mathcal{U}$ and their spatial derivatives, is given by

$$
\left(\begin{array}{c}
\eta \\
\eta_{\mathcal{U}}
\end{array}\right)=\frac{4 \pi}{\kappa} \sum_{n=0}^{\infty} \alpha_{(n)}\left(\begin{array}{c}
\frac{\delta H_{(n)}}{\delta \mathcal{J}} \\
\frac{\delta H_{(n)}}{\delta \mathcal{U}}
\end{array}\right),
$$

where the $\alpha_{(n)}$ are arbitrary constants. Therefore, replacing the solution (3.16) in (3.14) one can integrate the charge in the functional sense (taking the delta outside), obtaining

$$
Q=\sum_{n=0}^{\infty} \alpha_{(n)} H_{(n)} .
$$

Thus, the conserved charges in the higher spin theory are precisely given by a linear combination of the Hamiltonians of the mBoussinesq hierarchy. Indeed, using the transformation law (3.12), one can show the Hamiltonians $H_{(n)}^{I}$ are in involution with respect to the Dirac bracket (3.15)

$$
\left\{H_{(m)}^{I}, H_{(n)}^{J}\right\}^{\star}=0
$$

as expected.

In sum, all the relevant properties of the integrable mBoussinesq hierarchy described in section 2 are derived from spin-3 gravity endowed with the boundary conditions defined in eqs. (3.4), (3.5) and (3.8). Thus, the reduced phase space of spin-3 gravity and its boundary dynamics are equivalent to the ones of the mBoussinesq hierarchy. In particular, this provides an explicit one-to-one map between solutions of the integrable system at the boundary and solutions of the higher spin gravity theory in the bulk. 


\subsection{Highest weight gauge, Miura map and the Boussinesq hierarchy}

Asymptotic conditions for spin-3 gravity on $\mathrm{AdS}_{3}$ in the highest weight gauge were first given in refs. [31, 32], where it was shown that the asymptotic symmetries are spanned by two copies of the classical $W_{3}$ algebra with the Brown-Henneaux central charge. In what follows we consider the generalization introduced in refs. [35, 36], where the most general form of the Lagrange multipliers $a_{t}$, compatible with the $W_{3}$ symmetry, is allowed. This generalization has the important property that it accommodates black holes carrying non-trivial higher spin charges.

In this subsection we show that, with a particular gauge transformation, the auxiliary connection in the diagonal gauge (3.5) can be mapped to an auxiliary connection in the highest weight gauge, such that the Miura transformation in eq. (2.13), that relates the mBoussinesq with the Boussinesq hierarchies, is recovered from a purely geometric construction in the higher spin theory. The analysis is very close to the one in ref. [26], with the main difference that now the Lagrange multipliers $\zeta$ and $\zeta_{\mathcal{U}}$ depend on the dynamical fields according to eq. (3.8).

The angular components of the auxiliary connection in the highest weight gauge $\hat{a}$ is assumed to be of the form

$$
\hat{a}_{\varphi}=L_{1}-\frac{1}{2} \mathcal{L} L_{-1}-\frac{1}{4 \sqrt{3}} \mathcal{W} W_{-2} .
$$

Following $[35,36]$, the most general form of $\hat{a}_{t}$ which is compatible with the field equations is

$$
\begin{aligned}
\hat{a}_{t}= & \mu L_{1}-\frac{\sqrt{3}}{2} \nu W_{2}-\mu^{\prime} L_{0}+\frac{\sqrt{3}}{2} \nu^{\prime} W_{1}+\frac{1}{2}\left(\mu^{\prime \prime}-\mu \mathcal{L}-2 \mathcal{W} \nu\right) L_{-1} \\
& -\frac{\sqrt{3}}{48}\left(4 \mathcal{W} \mu-7 \mathcal{L}^{\prime} \nu^{\prime}-2 \nu \mathcal{L}^{\prime \prime}-8 \mathcal{L} \nu^{\prime \prime}+6 \mathcal{L}^{2} \nu+\nu^{\prime \prime \prime \prime}\right) W_{-2} \\
& -\frac{\sqrt{3}}{4}\left(\nu^{\prime \prime}-2 \mathcal{L} \nu\right) W_{0}+\frac{\sqrt{3}}{12}\left(\nu^{\prime \prime \prime}-2 \nu \mathcal{L}^{\prime}-5 \mathcal{L} \nu^{\prime}\right) W_{-1} .
\end{aligned}
$$

It is possible to find a gauge group element $g=g^{(1)} g^{(2)}$, such that the auxiliary connection in the diagonal gauge $a$ is mapped to the auxiliary connection in the highest weight gauge $\hat{a}$, by a gauge transformation of the form $\hat{a}=g^{-1}(d+a) g$, with

$$
\begin{aligned}
g^{(1)} & =\exp \left[x L_{1}+y W_{1}+z W_{2}\right] \\
g^{(2)} & =\exp \left[-\frac{1}{2} \mathcal{J} L_{-1}-\frac{\sqrt{3}}{6} \mathcal{U} W_{-1}+\frac{\sqrt{3}}{12}\left(\mathcal{J U}+\frac{1}{2} \mathcal{U}^{\prime}\right) W_{-2}\right] .
\end{aligned}
$$

Here, the functions $x, y, z$ are restricted to obey the following differential equations

$$
\begin{aligned}
& x^{\prime}=1+x \mathcal{J}+\sqrt{3} y \mathcal{U}, \\
& y^{\prime}=y \mathcal{J}+\sqrt{3} x \mathcal{U}, \\
& z^{\prime}=-\frac{1}{2} y+2 z \mathcal{J} .
\end{aligned}
$$


The fields $\mathcal{L}$ and $\mathcal{W}$ are then related to the fields $\mathcal{J}$ and $\mathcal{U}$ precisely by the Miura transformation (2.13)

$$
\begin{aligned}
\mathcal{L} & =\frac{1}{2} \mathcal{J}^{2}+\frac{1}{2} \mathcal{U}^{2}+\mathcal{J}^{\prime} \\
\mathcal{W} & =\frac{1}{3} \mathcal{U}^{3}-\mathcal{J}^{2} \mathcal{U}-\frac{1}{2} \mathcal{U} \mathcal{J}^{\prime}-\frac{3}{2} \mathcal{J} \mathcal{U}^{\prime}-\frac{1}{2} \mathcal{U}^{\prime \prime}
\end{aligned}
$$

The Lagrange multipliers in the highest weight gauge, given by $\mu$ and $\nu$, are related to the variables in the diagonal gauge through the following equations

$$
\begin{aligned}
\zeta & =\mathcal{J} \mu-\mu^{\prime}-2\left(\mathcal{J U}+\frac{1}{2} \mathcal{U}^{\prime}\right) \nu+\frac{1}{2} \mathcal{U} \nu^{\prime} \\
\zeta_{\mathcal{U}} & =\mathcal{U} \mu-\left(\mathcal{J}^{2}-\mathcal{U}^{2}-\mathcal{J}^{\prime}\right) \nu+\frac{3}{2} \mathcal{J} \nu^{\prime}-\frac{1}{2} \nu^{\prime \prime}
\end{aligned}
$$

The complete Boussinesq hierarchy is then obtained from the mBoussinesq one by virtue of the relations (3.19) and (3.20). Indeed, from (3.20), one can prove that the chemical potentials in the highest weight gauge take the form

$$
\mu=\frac{4 \pi}{\kappa} \frac{\delta H_{(k)}^{\mathrm{Bsq}}}{\delta \mathcal{L}}, \quad \nu=\frac{4 \pi}{\kappa} \frac{\delta H_{(k)}^{\mathrm{Bsq}}}{\delta \mathcal{W}}
$$

where $H_{(k)}^{\mathrm{Bsq}}$ corresponds the $k$-th Hamiltonian of the Boussinesq hierarchy (see appendix C for more details on the Boussinesq hierarchy). For example, for the first member given by $k=1$, one has

$$
H_{(1)}^{\mathrm{Bsq}}=\frac{\kappa}{4 \pi} \int d \phi\left(\lambda_{1} \mathcal{L}+\lambda_{2} \mathcal{W}\right)
$$

and hence the chemical potentials in the highest weight gauge become

$$
\mu=\lambda_{1}, \quad \nu=\lambda_{2}
$$

In the particular case with $\lambda_{1}=1$ and $\lambda_{2}=0$ (first flow), the equations of motion are given by two chiral movers, and the asymptotic conditions in eqs. (3.17), (3.18) reduce to the ones in refs. [31,32] but written in terms of the composite fields $\mathcal{L}$ and $\mathcal{W}$ according to (2.13). On the other hand, for the second flow with $\lambda_{1}=0$ and $\lambda_{2}=1$, the field equation in the bulk become equivalent to the Boussinesq equation in (2.14), in agreement with the result found in ref. [6]. 


\section{Black holes}

The line element is no longer gauge invariant in higher spin gravity, since it generically changes under the action of a higher spin gauge transformation. Therefore, the spacetime geometry and the causal structure cannot be directly used to define black holes. In refs. [37, 38], a new notion of higher spin black hole was introduced in the Euclidean formulation of the theory, by requiring trivial holonomies for the gauge connection around a thermal cycle $\mathcal{C}$, i.e.,

$$
\mathcal{H}_{\mathcal{C}}=\mathcal{P} e^{\int_{\mathcal{C}} a^{ \pm}}=1
$$

Here we have restored the \pm superscript to denote the plus/minus copy of the gauge field. If we assume that the Euclidean time is in the range $0 \leq t_{E}<1$ then, for time-independent configurations in (3.5), the regularity condition (4.1) imposes the following restrictions on $\zeta^{ \pm}$and $\zeta_{\mathcal{U}}^{ \pm}$

$$
\zeta^{ \pm}=\pi(2 n+m), \quad \zeta_{\mathcal{U}}^{ \pm}=\sqrt{3} \pi m,
$$

with $m$ and $n$ being integers. Static configurations that obey (4.2) are regular Euclidean solutions and consequently we call them "black holes."

According to ref. [26], the entropy takes the form

$$
S=\frac{\kappa}{4} \int d \phi\left((2 n+m)\left(\mathcal{J}^{+}+\mathcal{J}^{-}\right)+\sqrt{3} m\left(\mathcal{U}^{+}+\mathcal{U}^{-}\right)\right),
$$

which, by virtue of (3.8), (4.2) and (2.11), obeys the following first law

$$
\delta S=\sum_{I=1}^{2}\left(\lambda_{I}^{+} \delta H_{(k)}^{I+}+\lambda_{I}^{-} \delta H_{(k)}^{I-}\right),
$$

where $H_{(k)}^{I \pm}$ are the left/right $k$-th Hamiltonian of the mBoussinesq hierarchy associated to the flows labelled by $I=1,2$. Note that the constants $\lambda_{I}^{ \pm}$correspond to the chemical potentials conjugate to the extensive quantities $H_{(k)}^{I \pm}$.

When the integers $n$ and $m$ acquire the values $n=1, m=0$, we obtain a branch which is connected with the pure gravitational sector and the BTZ black hole. In that case, the entropy acquires the simple expression

$$
S=\frac{\kappa}{2} \int d \phi\left(\mathcal{J}^{+}+\mathcal{J}^{-}\right) .
$$

As was pointed out in [26], for constants $\mathcal{J}^{ \pm}$and $\mathcal{U}^{ \pm}$, the entropy for this branch acquires the expected form found in $[35,36]$ for a higher spin black hole, once it is written in terms of the charges of the $W$-algebra.

In sum, black holes that fit within our boundary conditions in eqs. (3.5) and (3.8) are identified with static solutions of the $k$-th element of the mBoussinesq hierarchy. It is worth noting that it is of fundamental importance to consider both flows to admit generic black hole configurations without restricting the possible space of solutions. To illustrate some of their properties, we will study the particular cases with $k=0,1,2$ in the branch connected with the BTZ black hole. For simplicity we consider only the plus copy. 
Case with $\boldsymbol{k}=\mathbf{0}$. The general solution of the field equations (3.10) that obeys (4.2) is given by two arbitrary functions of $\phi$, i.e., $\mathcal{J}=\mathcal{J}(\phi)$ and $\mathcal{U}=\mathcal{U}(\phi)$. This case corresponds to the "higher spin black flower" described in ref. [26], carrying an infinite set of $\hat{u}(1)$ soft hairy charges. The particular configuration with constant $\mathcal{J}$ and vanishing $\mathcal{U}$ in the branch with $n=1, m=0$, corresponds to the BTZ black hole embedded within this set of boundary conditions.

Case with $\boldsymbol{k}=\mathbf{1}$. The choice of boundary conditions (3.8), together with the regularity conditions, imply the following differential equations

$$
\begin{aligned}
\lambda_{1} \mathcal{J}-\lambda_{2}\left(\mathcal{U}^{\prime}+2 \mathcal{J U}\right) & =2 \pi, \\
\lambda_{1} \mathcal{U}+\lambda_{2}\left(\mathcal{J}^{\prime}-\mathcal{J}^{2}+\mathcal{U}^{2}\right) & =0 .
\end{aligned}
$$

These equations relate the chemical potentials $\lambda_{1}$ and $\lambda_{2}$ with the fields $\mathcal{J}$ and $\mathcal{U}$. In particular, for constants $\mathcal{J}$ and $\mathcal{U}$ we obtain

$$
\lambda_{1}=\frac{2 \pi\left(\mathcal{J}^{2}-\mathcal{U}^{2}\right)}{\mathcal{J}\left(\mathcal{J}^{2}-3 \mathcal{U}^{2}\right)}, \quad \lambda_{2}=\frac{2 \pi \mathcal{U}}{\mathcal{J}\left(\mathcal{J}^{2}-3 \mathcal{U}^{2}\right)}
$$

The regularity conditions for the BTZ solution in the pure gravity sector is recovered when $\mathcal{U}=0$, as expected. On the other hand, the configurations with $\mathcal{J}\left(\mathcal{J}^{2}-3 \mathcal{U}^{2}\right)=0$ possess non-trivial holonomies along the thermal cycle. Hence, one might identify them with extremal configurations, along the lines of ref. [39] (see also [40]). Indeed, when $\mathcal{U}^{ \pm}=0$, solutions with vanishing $\mathcal{J}^{+}$or $\mathcal{J}^{-}$correspond to extremal BTZ black holes.

Case with $\boldsymbol{k}=\mathbf{2}$. For constants $\mathcal{J}$ and $\mathcal{U}$, the regularity conditions (4.2) fix the chemical potentials $\lambda_{I}$ according to

$$
\begin{aligned}
\lambda_{1} & =\frac{3 \pi \mathcal{U}\left(15 \mathcal{J}^{4}-10 \mathcal{J}^{2} \mathcal{U}^{2}+7 \mathcal{U}^{4}\right)}{2 \mathcal{J}\left(9 \mathcal{J}^{8}-72 \mathcal{J}^{6} \mathcal{U}^{2}+210 \mathcal{J}^{4} \mathcal{U}^{4}-224 \mathcal{J}^{2} \mathcal{U}^{6}-3 \mathcal{U}^{8}\right)} \\
\lambda_{2} & =\frac{3 \pi\left(3 \mathcal{J}^{4}+6 \mathcal{J}^{2} \mathcal{U}^{2}-5 \mathcal{U}^{4}\right)}{2 \mathcal{J}\left(9 \mathcal{J}^{8}-72 \mathcal{J}^{6} \mathcal{U}^{2}+210 \mathcal{J}^{4} \mathcal{U}^{4}-224 \mathcal{J}^{2} \mathcal{U}^{6}-3 \mathcal{U}^{8}\right)}
\end{aligned}
$$

When $\mathcal{U}=0$, the auxiliary connection (3.5) reduces to the one that describes the BTZ geometry [5]. However, in contrast to the case with $k=1$, the chemical potential $\lambda_{1}$ associated to the first flow now vanishes, and hence, the information of the BTZ black hole is completely encoded in the second flow.

Note that for the cases with $k=1$ and $k=2$ described above, it is of fundamental importance to take into account both flows to have black holes characterized by two independent constants $\mathcal{J}^{ \pm}$and $\mathcal{U}^{ \pm}$for each copy. If one of the chemical potentials $\lambda_{1}^{ \pm}$or $\lambda_{2}^{ \pm}$ is set to zero, then eqs. (4.6) and (4.7) would imply non-trivial restrictions in the values of $\mathcal{J}^{ \pm}$and $\mathcal{U}^{ \pm}$, truncating in this way the spectrum of allowed black hole configurations. 


\section{$5 \quad$ Hamiltonian reduction and boundary dynamics}

In this section we will discuss the Hamiltonian reduction and the boundary dynamics of the Chern-Simons action that describes spin-3 gravity endowed with the boundary conditions associated to the mBoussinesq hierarchy. In the case of pure gravity with Brown-Henneaux asymptotic conditions, the boundary dynamics is described by two left and right chiral bosons which, by virtue of a Bäcklund transformation, turn out to be equivalent to a Liouville theory $[33,41]$. The analysis was done by performing a Hamiltonian reduction of the Wess-Zumino-Witten (WZW) theory at the boundary [42-45].

Here we follow an approach similar to the one proposed in ref. [46] for KdV-type boundary conditions in pure gravity, where instead of passing through the WZW theory, the boundary conditions are implemented directly in the Hamiltonian action (see also [47] for a different Hamiltonian reduction in the context of the KdV hierarchy).

Let us consider the Hamiltonian action with the appropriate boundary term in eq. (3.6). The constraints $F_{i j}=0$ are locally solved by expressing the spatial components of the gauge connection in terms of a group element $G$ as $A_{i}=G^{-1} \partial_{i} G$, provided that there are no holes in the spatial section. After replacing the solution of the constraints into the action (3.6), the following decomposition is obtained

$$
I_{\text {can }}[A]=I_{1}+I_{2}+B_{\infty},
$$

where

$$
\begin{aligned}
& I_{1}=\frac{\kappa}{16 \pi} \int d t d r d \phi \epsilon^{i j}\left\langle\partial_{t}\left(G^{-1}\right) \partial_{i} G G^{-1} \partial_{j} G\right\rangle, \\
& I_{2}=-\frac{\kappa}{16 \pi} \int d \phi d t\left\langle\partial_{t} G \partial_{\phi}\left(G^{-1}\right)\right\rangle .
\end{aligned}
$$

The Wess-Zumino term $I_{1}$ reduces to a boundary term that vanishes provided the group element is decomposed as $G=g(t, \phi) b(r)$ near the boundary (see appendix E for a detailed proof). Here $b(r)$ is the group element that depends on the radial coordinate in eq. (3.4), while $g(t, \phi)$ is such that the auxiliary connection $a$ in (3.5) is written as $a_{i}=g^{-1} \partial_{i} g$. With this decomposition the term $I_{2}$ becomes

$$
I_{2}=-\frac{\kappa}{16 \pi} \int d \phi d t\left\langle\dot{g} \partial_{\phi} g^{-1}\right\rangle .
$$

Since the auxiliary connection in (3.5) is a diagonal matrix, we can write the group element $g$ as follows

$$
g=\exp \left[\sqrt{\frac{8 \pi}{\kappa}} \varphi L_{0}+\sqrt{\frac{6 \pi}{\kappa}} \psi W_{0}\right],
$$

where $\varphi$ and $\psi$ are functions that depend only on $t$ and $\phi$. In what follows we will assume that these fields are periodic in the angle, and consequently possible contributions coming from non-trivial holonomies around $\phi$ are not considered in this analysis.

Consistency with the asymptotic form of the auxiliary connection (3.5) then implies

$$
\mathcal{J}=\sqrt{\frac{8 \pi}{\kappa}} \varphi^{\prime}, \quad \mathcal{U}=\sqrt{\frac{8 \pi}{\kappa}} \psi^{\prime} .
$$


Replacing (5.5) in (5.4) we find

$$
I_{2}=\int d \phi d t\left(\varphi^{\prime} \dot{\varphi}+\psi^{\prime} \dot{\psi}\right)
$$

Thus, if we use the expression (3.9) for the boundary term $B_{\infty}$, we finally obtain the following reduced action at the boundary

$$
I_{(k)}=\int d t\left[\int d \phi\left(\varphi^{\prime} \dot{\varphi}+\psi^{\prime} \dot{\psi}\right)-H_{(k)}\right] .
$$

This action describes the dynamics of the fields $\varphi$ and $\psi$, whose interactions are described by the $k$-th Hamiltonian of the mBoussinesq hierarchy. The members of the mBoussinesq hierarchy are then recovered from the equations of motion derived from the action (5.8), provided we identify the fields according to (5.6). In this sense, the field equations coming from (5.8) define "potential equations" for the ones of the mBoussinesq hierarchy.

The action (5.8) is invariant under the following transformations

$$
\delta \varphi=\sqrt{\frac{\kappa}{8 \pi}} \eta+f(t), \quad \delta \psi=\sqrt{\frac{\kappa}{8 \pi}} \eta_{\mathcal{U}}+f_{\mathcal{U}}(t)
$$

where the parameter $\eta$ and $\eta_{\mathcal{U}}$, given by (3.16), are associated to the infinite charges in involution of the integrable system. Indeed, all the Hamiltonians of the hierarchy may be obtained by a direct application of Noether theorem. On the other hand, the arbitrary functions of the time $f(t)$ and $f_{\mathcal{U}}(t)$ define gauge symmetries of (5.8) that allow to gauge away the zero modes of these fields.

Furthermore, the action (5.8) has an additional Lifschitz scaling symmetry in the particular case when only one of the two flows is considered. For the first flow, with $\lambda_{1}=1$ and $\lambda_{2}=0$, the dynamical exponent is $z=3 k-2$, while for the second flow, with $\lambda_{1}=0$ and $\lambda_{2}=1$, is $z=3 k-1$, as expected from the invariance properties of the mBoussinesq hierarchy described in section 2 . For the $I$-th flow of the $k$-th element of the hierarchy, the generators of the Lifshitz algebra are given by

$$
H=H_{(k)}^{I}, \quad P=H_{(1)}^{1}, \quad D=-\frac{\kappa}{4 \pi} \int d \phi\left(\frac{1}{2} \phi\left(\mathcal{J}^{2}+\mathcal{U}^{2}\right)\right)-z t H_{(k)}^{I} .
$$

Here, $H$ is the generators of translations in time, $P$ of translations in space, and $D$ of anisotropic dilatations. Using the Dirac brackets (3.15) it is straightforward to show that they close in the Lifshitz algebra

$$
\{P, H\}^{\star}=0, \quad\{D, P\}^{\star}=P, \quad\{D, H\}^{\star}=z H,
$$

where $z$ is the dynamical exponent associated to the corresponding flow.

Let us consider as an example the case with $k=1$. The action then takes the following form

$$
I_{(1)}=\int d \phi d t\left[\varphi^{\prime} \dot{\varphi}+\psi^{\prime} \dot{\psi}-\lambda_{1}\left(\varphi^{\prime 2}+\psi^{\prime 2}\right)+2 \lambda_{2}\left(\varphi^{\prime} \psi^{\prime \prime}+\sqrt{\frac{8 \pi}{\kappa}}\left(\varphi^{\prime 2} \psi^{\prime}-\frac{1}{3} \psi^{\prime 3}\right)\right)\right] .
$$

For the flow with $\lambda_{1}=1$ and $\lambda_{2}=0$ we recover the Floreanini-Jackiw action for two free chiral bosons [48]. On the other hand, for the flow with $\lambda_{1}=0$ and $\lambda_{2}=1$, the action contains non-trivial interacting term and is invariant under Lifshitz transformations with dynamical exponent $z=2$, as expected. 


\section{Some extensions: Generalized Gibbs ensemble, spin-N gravity and modified Gelfand-Dickey hierarchies}

\subsection{Generalized Gibbs Ensemble}

The existence of an infinite number of commuting charges in the mBoussinesq hierarchy opens the possibility of study more general thermodynamic ensembles that generically might include all possible charges. They are called "Generalized Gibbs Ensemble" (GGE).

In the case of a two-dimensional conformal field theory, a GGE is constructed with the infinite set of charges in involution of the KdV hierarchy, which are obtained as composite operators in terms of the stress tensor [49-51] (see refs. [6, 10, 11, 52-62] for recent results on GGE).

In our context, the Hamiltonians of the mBoussinesq hierarchy can be used to construct the GGE of a two-dimensional CFT with spin-3 currents. As discussed in subsection 3.5, the Miura transformation (2.13) maps the Hamiltonians of the mBoussinesq hierarchy into the Hamiltonians of the Boussinesq one, which depend on the stress tensor $\mathcal{L}$ and the spin-3 current $\mathcal{W}$. These Hamiltonians belong to the enveloping algebra of the $W_{3}$-algebra, and consequently define an infinite set of commuting charges that are composite operators in terms of $\mathcal{L}$ and $\mathcal{W}$. In this sense, the relation between the mBoussinesq hierarchy and spin-3 gravity discussed in this article, provides a natural holographic bulk dual description of this type of GGE.

This may be implemented as follows. Instead of considering one particular $H_{(k)}$ as the Hamiltonian of the dynamical system, we deal with a linear combination of them, i.e.,

$$
H_{\mathrm{GGE}}=\sum_{n=1}^{\infty} \sum_{I=1}^{2} \gamma_{n}\left(\lambda_{I} H_{(n)}^{I}\right)
$$

If we want to interpret this general Hamiltonian as the one of a $\mathrm{CFT}_{2}$ given by $H_{(1)}^{1}$, deformed by (multitrace) deformations that include spin-3 currents, then we must identify the inverse (right) temperature as $\beta_{+}=T_{+}^{-1}=\alpha_{1} \lambda_{1}$, and the additional chemical potentials as $\mu_{n, I}:=T_{+} \alpha_{n} \lambda_{I}$. However, this is not the only possibility. Any $H_{(k)}^{I}$ could be considered as the "undeformed Hamiltonian," allowing new branches that generically change the phase structure of the theory [63].

Black holes are described by static configurations of the dynamical system with Hamiltonian (6.1). The regularity condition (4.2) then takes the form

$$
\sum_{n=1}^{\infty} \sum_{I=1}^{2} \gamma_{n} \lambda_{I} \frac{\delta H_{(n)}^{I}}{\delta \mathcal{J}}=\frac{\kappa}{2}, \quad \sum_{n=1}^{\infty} \sum_{I=1}^{2} \gamma_{n} \lambda_{I} \frac{\delta H_{(n)}^{I}}{\delta \mathcal{U}}=0
$$

These equations guarantee that the Euclidean action principle attains an extremum, and hence they fully characterize the thermodynamics of the GGE.

On the other hand, the boundary dynamics obtained by the Hamiltonian reduction is easily generalized to the case when the Hamiltonian is given by (6.1). Indeed, the boundary action now becomes

$$
I_{\mathrm{GGE}}=\int d t\left[\int d \phi\left(\varphi^{\prime} \dot{\varphi}+\psi^{\prime} \dot{\psi}\right)-H_{\mathrm{GGE}}\right]
$$




\subsection{Higher spin gravity with gauge group $\mathrm{SL}(N, \mathbb{R}) \times \mathrm{SL}(N, \mathbb{R})$ and modified Gelfand-Dickey hierarchies}

Our results can be generalized to the case of three-dimensional higher spin gravity with gauge group $\mathrm{SL}(N, \mathbb{R}) \times \mathrm{SL}(N, \mathbb{R})$, where the corresponding integrable systems are the called "modified Generalized KdV hierarchies," or "modified Gelfand-Dickey (mGD) hierarchies" (see e.g. chapter 4 of ref. [64]). The link with higher spin gravity is based on the zero curvature formulation of these integrable systems [25].

Let us consider asymptotic conditions for spin- $N$ gravity described by the following auxiliary gauge connection valued on the $s l(N, \mathbb{R})$ algebra

$$
a=(\mathcal{J} d \phi+\zeta d t) L_{0}+\sum_{s=3}^{N} \sigma_{s}\left(\mathcal{U}^{(s)} d \phi+\zeta_{\mathcal{U}}^{(s)} d t\right) W_{0},
$$

with $\sigma_{s}$ given by

$$
\sigma_{s}=\left(\frac{(2 s-1) !(2 s-2) !}{48(s-1) !^{4}} \frac{1}{\prod_{i=2}^{s-1}\left(N^{2}-i^{2}\right)}\right)^{\frac{1}{2}} .
$$

The Chern-Simons action takes the same form as in (3.2), where one has to replace $\kappa_{3} \rightarrow$ $\kappa_{N}=3 l /\left(2 N\left(N^{2}-1\right) G\right)$. Hence, the variation of the boundary term of the canonical Chern-Simons action becomes

$$
\delta B_{\infty}=-\frac{\kappa}{4 \pi} \int d t d \phi\left(\zeta \delta \mathcal{J}+\sum_{s=3}^{N} \zeta_{\mathcal{U}}^{(s)} \delta \mathcal{U}^{(s)}\right) .
$$

To make contact with the mGD hierarchies, we choose the Lagrange multipliers as follows

$$
\zeta=\frac{4 \pi}{\kappa} \frac{\delta H_{(k, N)}^{\mathrm{mGD}}}{\delta \mathcal{J}}, \quad \zeta_{\mathcal{U}}^{(s)}=\frac{4 \pi}{\kappa} \frac{\delta H_{(k, N)}^{\mathrm{mGD}}}{\delta \mathcal{U}^{(s)}}
$$

where $H_{(k, N)}^{\mathrm{mGD}}$ corresponds to the $k$-th Hamiltonian of the $N$-th hierarchy [25]. With this choice of boundary conditions, the boundary term of the canonical Chern-Simons action integrates as

$$
B_{\infty}=-\int d t H_{(k, N)} .
$$

As expected, the Hamiltonian of the higher spin theory coincides with one of the hierarchy.

The Dirac brackets are described by $N-1 \hat{u}(1)$ current algebras

$$
\begin{gathered}
\left\{\mathcal{J}(\phi), \mathcal{J}\left(\phi^{\prime}\right)\right\}^{\star}=\frac{4 \pi}{\kappa} \partial_{\phi} \delta\left(\phi-\phi^{\prime}\right), \\
\left\{\mathcal{U}^{(s)}(\phi), \mathcal{U}^{\left(s^{\prime}\right)}\left(\phi^{\prime}\right)\right\}^{\star}=\frac{4 \pi}{\kappa} \partial_{\phi} \delta\left(\phi-\phi^{\prime}\right) \delta^{s, s^{\prime}},
\end{gathered}
$$

matching the first Poisson structure of the mGD hierarchies. Moreover, their members are obtained from the equations of motion of the higher spin theory with the boundary conditions (6.3), (6.5), and are given by

$$
\dot{\mathcal{J}}=\frac{4 \pi}{\kappa} \partial_{\phi}\left(\frac{\delta H_{(k, N)}^{\mathrm{mGD}}}{\delta \mathcal{J}}\right), \quad \dot{\mathcal{U}}^{(s)}=\frac{4 \pi}{\kappa} \partial_{\phi}\left(\frac{\delta H_{(k, N)}^{\mathrm{mGD}}}{\delta \mathcal{U}^{(s)}}\right) .
$$


The mGD hierarchies are related to the called "Generalized KdV hierarchies," or "Gelfand-Dickey (GD) hierarchies" by an appropriate generalization of the Miura transformation. One of the two Poisson brackets of the GD hierarchies is described by the $W_{N}$-algebra, whose generators are composite in terms of the $\hat{u}(1)$ currents of the mGD hierarchies. Hence, according to the Hamiltonian reduction in [25], the generalized Miura transformation should emerge geometrically from our boundary conditions once they are expressed in the highest weight gauge, as in the case for $N=3$ described in subsection 3.5. Note that since generically the expression for the stress tensor of the $W_{N}$-algebra in terms of $\hat{u}(1)$ currents is the one of a twisted Sugawara construction, there is a particular flow in which the currents are chiral. This case precisely corresponds to one of the proposals in ref. [9], for describing gravitational duals of averaged CFT's on the Narain lattice [65, 66] (see [67] for an alternative proposal for a possible gravitational dual).

\section{$7 \quad$ Final remarks}

We have shown that the dynamics of three-dimensional higher spin gravity with gauge group $S L(N, \mathbb{R}) \times S L(N, \mathbb{R})$ endowed with a certain special class of boundary conditions in the diagonal gauge, reduces to the one of the integrable system corresponding to the $N$ th modified Gelfand-Dickey hierarchy. The particular case with $N=3$ is associated to the mBoussinesq hierarchy, whose first member defines a potential equation for the Boussinesq one, which was found for the first time in the nineteenth century in the context of the study of solitary waves (solitons) in fluid dynamics. In this sense, it would be interesting to explore the possibility that these results could be understood in the context of the fluid-gravity correspondence [68-71], along the lines of [72].

On the other hand, in section 5 we studied the Hamiltonian reduction and the boundary dynamics induced by our choice of boundary conditions. In this analysis it was explicitly assumed that the fields $\varphi$ and $\psi$ were periodic in the angle $\phi$, leading to a dynamics described by the action (5.8). However, this assumption does not take into account the black hole configurations described in section 4. This suggests the possibility of trying to introduce non-trivial holonomies around the non-contractible cycle along the lines of ref. [73], to incorporate these solutions into the analysis.

The entire integrable structure of the family of $(\mathrm{m})$ GD hierarchies can be embedded in a single universal (2+1)-dimensional integrable system called the Kadomtsev-Petviashvili (KP) hierarchy [74]. Many different (1+1)-dimensional integrable systems, including the (m)GD hierarchies, are recovered for some specific (dimensional) reductions of the KP hierarchy (see e.g. [64]). It would then be natural to explore the possibility that the KP hierarchy could emerge from the asymptotic structure of a higher dimensional bulk (higher spin) gravitational theory.

Other extensions of our results might include the possible relation of certain twodimensional integrable systems with three-dimensional higher spin gravity with vanishing cosmological constant [75-79], generalizations in the context of hypergravity [39, 80-82], or extensions to generalized Boussinesq hierarchies as the ones described in ref. [83]. 


\section{Acknowledgments}

We thank Fábio Novaes and Ricardo Troncoso for some useful discussions. The work of EO was funded by the PhD grant CONICYT-PCHA/Doctorado Nacional/2016-21161352. This research has been partially supported by Fondecyt grants № 1171162, 1181496, 1181031. The Centro de Estudios Científicos (CECs) is funded by the Chilean Government through the Centers of Excellence Base Financing Program of Conicyt.

\section{A Second Hamiltonian structure of the modified Boussinesq hierarchy}

The second Hamiltonian structure of the mBoussinesq hierarchy is defined by the operator $\mathcal{D}_{(2)}$ in (2.6), whose explicit components are given by

$$
\begin{aligned}
\frac{\hat{\kappa}}{4 \pi} \mathcal{D}_{(2)}^{11}= & -4\left(2\left(\mathcal{U}^{\prime \prime}+2(\mathcal{J U})^{\prime}\right) \partial_{\phi}^{-1}\left(\mathcal{J} \partial_{\phi}+\partial_{\phi}^{2}\right)+\mathcal{J}^{\prime} \partial_{\phi}^{-1}\left(\left(4 \mathcal{J} \mathcal{U}+3 \mathcal{U}^{\prime}\right) \partial_{\phi}+\mathcal{U} \partial_{\phi}^{2}\right)\right. \\
& \left.+\left(8 \mathcal{J}^{2} \mathcal{U}-5 \mathcal{U} \mathcal{J}^{\prime}-3 \mathcal{U}^{\prime \prime}\right) \partial_{\phi}-3 \mathcal{U}^{\prime} \partial_{\phi}^{2}-2 \mathcal{U} \partial_{\phi}^{3}\right) \\
\frac{\hat{\kappa}}{4 \pi} \mathcal{D}_{(2)}^{12}= & -4\left(2\left(\mathcal{U}^{\prime \prime}+2(\mathcal{J} \mathcal{U})^{\prime}\right) \partial_{\phi}^{-1}\left(\mathcal{U} \partial_{\phi}\right)+\mathcal{J}^{\prime} \partial_{\phi}^{-1}\left(\left(2 \mathcal{J}^{2}-2 \mathcal{U}^{2}+\mathcal{J}^{\prime}\right) \partial_{\phi}+3 \mathcal{J} \partial_{\phi}^{2}+\partial_{\phi}^{3}\right)\right. \\
& \left.+\left(2 \mathcal{J}^{3}+2 \mathcal{J U}^{2}+2 \mathcal{U}^{2 \prime}-3 \mathcal{J}^{\prime}-\mathcal{J}^{\prime \prime}\right) \partial_{\phi}+\left(\mathcal{J}^{2}+\mathcal{U}^{2}-4 \mathcal{J}^{\prime}\right) \partial_{\phi}^{2}-2 \mathcal{J} \partial_{\phi}^{3}-\partial_{\phi}^{4}\right), \\
\frac{\hat{\kappa}}{4 \pi} \mathcal{D}_{(2)}^{21}= & -4\left(2\left(\mathcal{J}^{2 \prime}-\mathcal{U}^{2 \prime}-\mathcal{J}^{\prime \prime}\right) \partial_{\phi}^{-1}\left(\mathcal{J} \partial_{\phi}+\partial_{\phi}^{2}\right)+\mathcal{U}^{\prime} \partial_{\phi}^{-1}\left(\left(4 \mathcal{J} \mathcal{U}+3 \mathcal{U}^{\prime}\right) \partial_{\phi}+\mathcal{U} \partial_{\phi}^{2}\right)\right. \\
& \left.+\left(2 \mathcal{J}^{3}+2 \mathcal{J U}^{2}-4 \mathcal{J}^{2 \prime}+3 \mathcal{U} \mathcal{U}^{\prime}+\mathcal{J}^{\prime \prime}\right) \partial_{\phi}-\left(3 \mathcal{J}^{\prime}+\mathcal{J}^{2}+\mathcal{U}^{2}\right) \partial_{\phi}^{2}-2 \mathcal{J} \partial_{\phi}^{3}+\partial_{\phi}^{4}\right), \\
\frac{\hat{\kappa}}{4 \pi} \mathcal{D}_{(2)}^{22}= & -4\left(-2\left(\mathcal{J}^{\prime \prime}+\mathcal{U}^{2 \prime}-\mathcal{J}^{2 \prime}\right) \partial_{\phi}^{-1}\left(\mathcal{U} \partial_{\phi}\right)+\mathcal{U}^{\prime} \partial_{\phi}^{-1}\left(\left(2 \mathcal{J}^{2}-2 \mathcal{U}^{2}+\mathcal{J}^{\prime}\right) \partial_{\phi}+3 \mathcal{J} \partial_{\phi}^{2}+\partial_{\phi}^{3}\right)\right. \\
& \left.+\left(-4 \mathcal{U}^{3}+4 \mathcal{J}^{2} \mathcal{U}-3 \mathcal{J U}^{\prime}-4 \mathcal{J}^{\prime} \mathcal{U}+\mathcal{U}^{\prime \prime}\right) \partial_{\phi}+2 \mathcal{U}^{\prime} \partial_{\phi}^{2}+2 \mathcal{U} \partial_{\phi}^{3}\right) .
\end{aligned}
$$

\section{B Gelfand-Dickey polynomials and Hamiltonians of the modified Boussi- nesq hierarchy}

In this appendix we exhibit the first Gelfand-Dickey polynomials and Hamiltonians of the mBoussinesq hierarchy.

The Gelfand-Dickey polynomials can be explicitly constructed using the recurrence relation (2.9). The first of them are given by

$$
\begin{aligned}
\frac{4 \pi}{\hat{\kappa}} R_{\mathcal{J}}^{(0)}= & \lambda_{1}, \\
\frac{4 \pi}{\hat{\kappa}} R_{\mathcal{J}}^{(1)}= & \lambda_{1} \mathcal{J}-\lambda_{2}\left(\mathcal{U}^{\prime}+2 \mathcal{J} \mathcal{U}\right), \\
\frac{4 \pi}{\hat{\kappa}} R_{\mathcal{J}}^{(2)}= & 4 \lambda_{1}\left(-4 \mathcal{J}^{3} \mathcal{U}-\frac{4}{3} \mathcal{J} \mathcal{U}^{3}-2 \mathcal{J}^{2} \mathcal{U}^{\prime}-2 \mathcal{U}^{2} \mathcal{U}^{\prime}+2 \mathcal{J}^{\prime} \mathcal{U}^{\prime}+2 \mathcal{J}^{\prime \prime} \mathcal{U}+2 \mathcal{J} \mathcal{U}^{\prime \prime}+\mathcal{U}^{\prime \prime \prime}\right) \\
& +4 \lambda_{2}\left(\mathcal{J}^{5}+10 \mathcal{J}^{3} \mathcal{U}^{2}-\frac{5}{3} \mathcal{J U}^{4}-5 \mathcal{J} \mathcal{J}^{\prime 2}+5 \mathcal{J}^{2} \mathcal{U}^{2 \prime}-\frac{10}{12} \mathcal{U}^{4 \prime}\right. \\
& \left.\quad-5 \mathcal{J}^{\prime} \mathcal{U}^{2 \prime}+5 \mathcal{J U}^{\prime 2}-5 \mathcal{J}^{2} \mathcal{J}^{\prime \prime}-5 \mathcal{J}^{\prime \prime} \mathcal{U}^{2}-5 \mathcal{J}^{\prime} \mathcal{J}^{\prime \prime}+5 \mathcal{U}^{\prime} \mathcal{U}^{\prime \prime}+\mathcal{J}^{\prime \prime \prime \prime}\right),
\end{aligned}
$$




$$
\begin{aligned}
\frac{4 \pi}{\hat{\kappa}} R_{\mathcal{U}}^{(0)}= & \lambda_{2}, \\
\frac{4 \pi}{\hat{\kappa}} R_{\mathcal{U}}^{(1)}= & \lambda_{1} \mathcal{U}+\lambda_{2}\left(\mathcal{J}^{\prime}-\mathcal{J}^{2}+\mathcal{U}^{2}\right) \\
\frac{4 \pi}{\hat{\kappa}} R_{\mathcal{U}}^{(2)}= & 4 \lambda_{1}\left(-\mathcal{J}^{4}-2 \mathcal{J}^{2} \mathcal{U}^{2}+\frac{5}{3} \mathcal{U}^{4}+\frac{2}{3} \mathcal{J}^{3 \prime}+2 \mathcal{U}^{2} \mathcal{J}^{\prime}+\mathcal{J}^{\prime 2}-\mathcal{U}^{\prime 2}+2 \mathcal{J}^{\prime \prime} 2 \mathcal{U} \mathcal{U}^{\prime \prime}-\mathcal{J}^{\prime \prime \prime}\right) \\
& +4 \lambda_{2}\left(5 \mathcal{J}^{4} \mathcal{U}-\frac{10}{3} \mathcal{J}^{2} \mathcal{U}^{3}+\frac{7}{3} \mathcal{U}^{5}-\frac{10}{3} \mathcal{J}^{3 \prime} \mathcal{U}+\frac{10}{3} \mathcal{J}^{\prime} \mathcal{U}^{3}\right. \\
& \left.+5 \mathcal{U} \mathcal{J}^{\prime 2}-5 \mathcal{J}^{2 \prime} \mathcal{U}^{\prime}-5 \mathcal{U} \mathcal{U}^{\prime 2}+5 \mathcal{J}^{\prime \prime} \mathcal{U}^{\prime}-5 \mathcal{J}^{2} \mathcal{U}^{\prime \prime}-5 \mathcal{U}^{2} \mathcal{U}^{\prime \prime}+5 \mathcal{J}^{\prime} \mathcal{U}^{\prime \prime}+\mathcal{U}^{\prime \prime \prime \prime}\right)
\end{aligned}
$$

The corresponding Hamiltonians can then be obtained using eq. (2.10). Thus,

$$
\begin{aligned}
\frac{4 \pi}{\hat{\kappa}} H_{(0)}= & \int d \phi\left(\lambda_{1} \mathcal{J}+\lambda_{2} \mathcal{U}\right) \\
\frac{4 \pi}{\hat{\kappa}} H_{(1)}= & \int d \phi\left\{\frac{\lambda_{1}}{2}\left(\mathcal{J}^{2}+\mathcal{U}^{2}\right)+\lambda_{2}\left(\frac{1}{3} \mathcal{U}^{3}-\mathcal{J}^{2} \mathcal{U}-\mathcal{J} \mathcal{U}^{\prime}\right)\right\} \\
\frac{4 \pi}{\hat{\kappa}} H_{(2)}= & \int d \phi\left\{\frac { 4 \lambda _ { 1 } } { 3 } \left(\mathcal{U}^{5}-3 \mathcal{J}^{4} \mathcal{U}-2 \mathcal{J}^{2} \mathcal{U}^{3}-3 \mathcal{J}^{\prime 2} \mathcal{U}-2 \mathcal{J}^{3} \mathcal{U}^{\prime}-2 \mathcal{J} \mathcal{U}^{3 \prime}\right.\right. \\
& \left.+3 \mathcal{J}^{2} \mathcal{U}^{\prime \prime}-\frac{3}{2} \mathcal{U}^{2} \mathcal{U}^{\prime \prime}+3 \mathcal{J} \mathcal{U}^{\prime \prime \prime}\right)+\frac{2 \lambda_{2}}{3}\left(\mathcal{J}^{6}+15 \mathcal{J}^{4} \mathcal{U}^{2}-5 \mathcal{J}^{2} \mathcal{U}^{4}+\frac{7}{3} \mathcal{U}^{6}\right. \\
& +15 \mathcal{J}^{2} \mathcal{J}^{\prime 2}+15 \mathcal{U}^{2} \mathcal{J}^{\prime 2}+10 \mathcal{J}^{3} \mathcal{U}^{2 \prime}-5 \mathcal{J U}^{4 \prime}+15 \mathcal{J}^{2} \mathcal{U}^{\prime 2}+15 \mathcal{U}^{2} \mathcal{U}^{\prime 2} \\
& \left.\left.-10 \mathcal{J} \mathcal{J}^{\prime} \mathcal{J}^{\prime \prime}+30 \mathcal{J} \mathcal{U}^{\prime} \mathcal{U}^{\prime \prime}+3 \mathcal{J} \mathcal{J}^{\prime \prime \prime \prime}+3 \mathcal{U} \mathcal{U}^{\prime \prime \prime \prime}\right)\right\}
\end{aligned}
$$

\section{Boussinesq hierarchy}

The Boussinesq hierarchy is an integrable bi-Hamiltonian system which possesses two different Poisson brackets defined by the following operators

$$
\begin{aligned}
& \mathcal{D}_{(1)}^{\mathrm{Bsq}}=\frac{\pi}{2 \hat{\kappa}}\left(\begin{array}{cc}
0 & \partial_{\phi} \\
\partial_{\phi} & 0
\end{array}\right), \\
& \mathcal{D}_{(2)}^{\mathrm{Bsq}}=\frac{4 \pi}{\hat{\kappa}}\left(\begin{array}{cc}
2 \mathcal{L} \partial_{\phi}+\mathcal{L}^{\prime}-\partial_{\phi}^{3} & 3 \mathcal{W} \partial_{\phi}+2 \mathcal{W} \\
3 \mathcal{W} \partial_{\phi}+\mathcal{W}^{\prime} & -\frac{1}{2} \mathcal{L}^{\prime \prime \prime}+2 \mathcal{L}^{2 \prime}-\frac{9}{4}\left(\mathcal{L}^{\prime \prime}-\frac{16}{9} \mathcal{L}^{2}\right) \partial_{\phi}-\frac{15}{4} \mathcal{L}^{\prime} \partial_{\phi}^{2}-\frac{5}{2} \mathcal{L} \partial_{\phi}^{3}+\frac{1}{4} \partial_{\phi}^{5}
\end{array}\right) .
\end{aligned}
$$

The Poisson bracket associated to the operator $\mathcal{D}_{(2)}^{\text {Bsq }}$ is given by the classical $W_{3}$-algebra.

The infinite Hamiltonians in involution can be obtained using the following recursion relation

$$
\mathcal{D}_{(1)}^{\mathrm{Bsq}}\left(\begin{array}{c}
R_{\mathcal{L}} \\
R_{\mathcal{W}}
\end{array}\right)_{(k+1)}=\mathcal{D}_{(2)}^{\mathrm{Bsq}}\left(\begin{array}{c}
R_{\mathcal{L}} \\
R_{\mathcal{W}}
\end{array}\right)_{(k)} .
$$

Here the corresponding Gelfand-Dickey polynomials are defined through

$$
\left(\begin{array}{l}
R_{\mathcal{L}} \\
R_{\mathcal{W}}
\end{array}\right)_{(k)}=\left(\begin{array}{c}
\frac{\delta H_{(k)}^{\mathrm{Bsq}}}{\delta \mathcal{L}} \\
\frac{\delta H_{(k)}^{\mathrm{Bsq}}}{\delta \mathcal{W}}
\end{array}\right),
$$


where the first Hamiltonian is given by

$$
H_{(1)}^{\mathrm{Bsq}}=\frac{\hat{\kappa}}{4 \pi} \int d \phi\left(\lambda_{1} \mathcal{L}+\lambda_{2} \mathcal{W}\right)
$$

Therefore, the members of the hierarchy can be written as follows

$$
\left(\begin{array}{c}
\dot{\mathcal{L}} \\
\dot{\mathcal{W}}
\end{array}\right)_{(k)}=\mathcal{D}_{(1)}^{\mathrm{Bsq}}\left(\begin{array}{c}
R_{\mathcal{L}} \\
R_{\mathcal{W}}
\end{array}\right)_{(k+1)}=\mathcal{D}_{(2)}^{\mathrm{Bsq}}\left(\begin{array}{c}
R_{\mathcal{L}} \\
R_{\mathcal{W}}
\end{array}\right)_{(k)}
$$

As explained in section 2, the Boussinesq and the mBoussinesq hierarchies are related by the Miura transformation (2.13), that can be rewritten in the following vector form

$$
\left(\begin{array}{c}
\mathcal{L} \\
\mathcal{W}
\end{array}\right)=F[\mathcal{J}, \mathcal{U}]
$$

for a functional $F$ defined through (2.13). Taking the derivative with respect to the time one obtains

$$
\left(\begin{array}{c}
\dot{\mathcal{L}} \\
\dot{\mathcal{W}}
\end{array}\right)=M\left(\begin{array}{c}
\dot{\mathcal{J}} \\
\dot{\mathcal{U}}
\end{array}\right)
$$

where $M=M[\mathcal{J}, \mathcal{U}]$ correspond to the Fréchet derivative of $F$ with respect to $\mathcal{J}$ and $\mathcal{U}[27]$, and is precisely given by the matrix $M$ in (2.7), i.e.,

$$
M=\left(\begin{array}{cc}
\mathcal{J}+\partial_{\phi} & \mathcal{U} \\
-2 \mathcal{J U}-\frac{1}{2} \mathcal{U} \partial_{\phi}-\frac{3}{2} \mathcal{U}^{\prime} & \mathcal{U}^{2}-\mathcal{J}^{2}-\frac{1}{2} \mathcal{J}^{\prime}-\frac{3}{2} \mathcal{J} \partial_{\phi}-\frac{1}{2} \partial_{\phi}^{2}
\end{array}\right)
$$

If one takes into account its formal adjoint

$$
M^{\dagger}=\left(\begin{array}{cc}
\mathcal{J}-\partial_{\phi} & -2 \mathcal{J U}+\frac{1}{2} \mathcal{U} \partial_{\phi}-\mathcal{U}^{\prime} \\
\mathcal{U} & \mathcal{U}^{2}-\mathcal{J}^{2}+\mathcal{J}^{\prime}+\frac{3}{2} \mathcal{J} \partial_{\phi}-\frac{1}{2} \partial_{\phi}^{2}
\end{array}\right),
$$

the Gelfand-Dickey polynomials of both hierarchies are then related by

$$
\left(\begin{array}{l}
R_{\mathcal{J}} \\
R_{\mathcal{U}}
\end{array}\right)=M^{\dagger}\left(\begin{array}{c}
R_{\mathcal{L}} \\
R_{\mathcal{W}}
\end{array}\right)
$$

Taking into account eqs. (C.3), (3.11) and (C.4) we can write

$$
\left(\begin{array}{c}
\dot{\mathcal{L}} \\
\dot{\mathcal{W}}
\end{array}\right)_{(k)}=M \mathcal{D}\left(\begin{array}{c}
R_{\mathcal{J}} \\
R_{\mathcal{U}}
\end{array}\right)_{(k)}=M \mathcal{D} M^{\dagger}\left(\begin{array}{c}
R_{\mathcal{L}} \\
R_{\mathcal{W}}
\end{array}\right)_{(k)}=\mathcal{D}_{(2)}^{\mathrm{Bsq}}\left(\begin{array}{c}
R_{\mathcal{L}} \\
R_{\mathcal{W}}
\end{array}\right)_{(k)},
$$

which imply that the second Poisson structure for the Boussinesq hierarchy can be expressed in terms of the first Poisson structure of the mBoussinesq hierarchy according to

$$
\mathcal{D}_{(2)}^{\mathrm{Bsq}}=M \mathcal{D} M^{\dagger}
$$




\section{Fundamental representation of the principal embedding of $\operatorname{sl}(2, \mathbb{R})$ within $\operatorname{sl}(N, \mathbb{R})$}

In the principal embedding of the $\operatorname{sl}(2, \mathbb{R})$ algebra within the $\operatorname{sl}(N, \mathbb{R})$ algebra the generators can be written in the basis $\left\{L_{i}, W_{m}^{(s)}\right\}$, with $i=-1,0,1, s=3,4, \ldots$ and $m=$ $-s+1, \ldots, s-1$. In the fundamental representation of $s l(N, \mathbb{R})$, the generators may be represented by the following $N \times N$ matrices

$$
\begin{gathered}
\left(L_{1}\right)_{j k}=-\sqrt{j(N-j)} \delta_{j+1, k}, \\
\left(L_{-1}\right)_{j k}=\sqrt{k(N-k)} \delta_{j, k+1}, \\
\left(L_{0}\right)_{j k}=\frac{1}{2}(N+1-2 j) \delta_{j, k}, \\
W_{m}^{(s)}=2(-1)^{s-m-1} \frac{(s+m-1) !}{(2 s-2) !} \underbrace{\left[L_{-1},\left[L_{-1}, \cdots\left[L_{-1},\left(L_{1}\right)^{s-1}\right] \cdots\right]\right]}_{s-m-1 \text { terms }}, \\
=2(-1)^{s-m-1} \frac{(s+m-1) !}{(2 s-2) !}\left(\operatorname{ad}_{L_{-1}}\right)^{s-m-1}\left(L_{1}\right)^{s-1} .
\end{gathered}
$$

with $j, k=1, \ldots, N$, and where $\operatorname{ad}_{\mathrm{x}}(\mathrm{Y}):=[\mathrm{X}, \mathrm{Y}]$. From the commutation relations

$$
\begin{aligned}
{\left[L_{i}, L_{j}\right] } & =(i-j) L_{i+j}, \\
{\left[L_{i}, W_{m}^{(s)}\right] } & =((s-1) i-m) W_{i+m}^{(s)},
\end{aligned}
$$

it can be seen that the $L_{i}$ generators close in a $s l(2, \mathbb{R})$ subalgebra, while the generators $W_{m}^{(s)}$ transform in a spin- $s$ representation under $s l(2, \mathbb{R})$.

\section{E Wess-Zumino term}

Here we show that for our boundary conditions in (3.4), (3.5), (3.8), the Wess-Zumino term

$$
I_{1}=\frac{\kappa}{16 \pi} \int d t d r d \phi \epsilon^{i j}\left\langle\partial_{t}\left(G^{-1}\right) \partial_{i} G G^{-1} \partial_{j} G\right\rangle,
$$

in (5.2) vanishes.

Let us perform the following Gauss decomposition of the group element

$$
G=e^{T L_{1}+M W_{1}+Q W_{2}} e^{\Phi L_{0}+\Phi_{W} W_{0}} e^{X L_{-1}+Y W_{-1}+Z W_{-2}},
$$

where all the functions that appear in (E.1) generically depend on $t, r$ and $\phi$. Then, if we replace (E.1) in $I_{1}$, one can show that it reduces to a boundary term of the form

$$
\begin{aligned}
I_{1}= & \frac{\kappa}{16 \pi} \int d \phi d t\left[2 e^{\Phi+2 \Phi_{W}}\left(\left(X^{\prime}+Y^{\prime}\right)(\dot{M}+\dot{T})-(\dot{X}+\dot{Y})\left(M^{\prime}+T^{\prime}\right)\right)\right. \\
& -2 e^{\Phi-2 \Phi_{W}}\left(\left(X^{\prime}-Y^{\prime}\right)(\dot{M}-\dot{T})-(\dot{X}-\dot{Y})\left(M^{\prime}-T^{\prime}\right)\right) \\
& +e^{2 \Phi}\left(8\left(X Y^{\prime}-Y X^{\prime}\right) \dot{Q}-8(X \dot{Y}-Y \dot{X}) Q^{\prime}+8(T \dot{M}-M \dot{T}) Z^{\prime}-8\left(T M^{\prime}-M T^{\prime}\right) \dot{Z}\right. \\
& \left.\left.+4(X \dot{Y}-Y \dot{X})\left(T M^{\prime}-M T^{\prime}\right)-4\left(X Y^{\prime}-Y X^{\prime}\right)(T \dot{M}-M \dot{T})+16\left(Q^{\prime} \dot{Z}-Z^{\prime} \dot{Q}\right)\right)\right] .
\end{aligned}
$$


Now, it is useful to perform the following decomposition in the asymptotic region which is compatible with (3.4)

$$
G=g(t, \phi) b(r)
$$

with

$$
g(t, \phi)=\exp \left[\sqrt{\frac{8 \pi}{\kappa}} \varphi L_{0}+\sqrt{\frac{6 \pi}{\kappa}} \psi W_{0}\right],
$$

and where $b(r)$ is an arbitrary gauge group element depending on the radial coordinate that generically can be decomposed as

$$
b(r)=b_{(+)} b_{(0)} b_{(-)},
$$

with

$$
b_{(+)}=e^{\left(b_{1} L_{1}+\bar{b}_{1} W_{1}+\bar{b}_{2} W_{2}\right)}, \quad b_{(0)}=e^{\left(b_{0} L_{0}+\bar{b}_{0} W_{0}\right)}, \quad b_{(-)}=e^{\left(b_{-1} L_{-1}+\bar{b}_{-1} W_{-1}+\bar{b}_{-2} W_{-2}\right)} .
$$

Consistency with (E.1) then implies the following conditions

$$
\begin{array}{rlrl}
\Phi & =b_{0}(r)+\sqrt{\frac{8 \pi}{\kappa}} \varphi(t, \phi), & \Phi_{W} & =\bar{b}_{0}(r)+\sqrt{\frac{6 \pi}{\kappa}} \psi(t, \phi), \\
X & =b_{-1}(r), \\
Y & =e^{-2 \sqrt{\frac{8 \pi}{\kappa}} \varphi} \bar{b}_{-2}(r), \quad Z=\bar{b}_{-2}(r), \\
T & =e^{-\sqrt{\frac{8 \pi}{\kappa}} \varphi}\left(b_{1}(r) \cosh \left(2 \sqrt{\frac{6 \pi}{\kappa}} \psi\right)-\bar{b}_{1}(r) \sinh \left(2 \sqrt{\frac{8 \pi}{\kappa}} \varphi\left(\bar{b}_{1}(r) \cosh \left(2 \sqrt{\frac{6 \pi}{\kappa}} \psi\right)-b_{1}(r) \sinh \left(2 \sqrt{\frac{6 \pi}{\kappa}} \psi\right)\right),\right.\right.
\end{array}
$$

Note that since, $X, Y$ and $Z$ depend only on the radial coordinate, then the WZ term in eq. (E.2) identically vanishes.

Open Access. This article is distributed under the terms of the Creative Commons Attribution License (CC-BY 4.0), which permits any use, distribution and reproduction in any medium, provided the original author(s) and source are credited.

\section{References}

[1] J. Brown and M. Henneaux, Central Charges in the Canonical Realization of Asymptotic Symmetries: An Example from Three-Dimensional Gravity, Commun. Math. Phys. 104 (1986) 207 [INSPIRE].

[2] G. Compère, W. Song and A. Strominger, New Boundary Conditions for AdS3, JHEP 05 (2013) 152 [arXiv: 1303.2662] [INSPIRE].

[3] C. Troessaert, Enhanced asymptotic symmetry algebra of AdS $S_{3}$, JHEP 08 (2013) 044 [arXiv: 1303.3296] [INSPIRE].

[4] S.G. Avery, R.R. Poojary and N.V. Suryanarayana, $\operatorname{An~sl}(2, \mathbb{R})$ current algebra from $A d S_{3}$ gravity, JHEP 01 (2014) 144 [arXiv:1304.4252] [INSPIRE].

[5] H. Afshar et al., Soft Heisenberg hair on black holes in three dimensions, Phys. Rev. D 93 (2016) 101503 [arXiv:1603.04824] [INSPIRE]. 
[6] A. Pérez, D. Tempo and R. Troncoso, Boundary conditions for General Relativity on $A d S_{3}$ and the KdV hierarchy, JHEP 06 (2016) 103 [arXiv: 1605.04490] [INSPIRE].

[7] D. Grumiller and M. Riegler, Most general AdS $S_{3}$ boundary conditions, JHEP 10 (2016) 023 [arXiv: 1608.01308] [INSPIRE].

[8] E. Ojeda and A. Pérez, Boundary conditions for General Relativity in three-dimensional spacetimes, integrable systems and the KdV/mKdV hierarchies, JHEP 08 (2019) 079 [arXiv: 1906.11226] [INSPIRE].

[9] A. Pérez and R. Troncoso, Gravitational dual of averaged free CFT's over the Narain lattice, JHEP 11 (2020) 015 [arXiv:2006.08216] [INSPIRE].

[10] J. de Boer and D. Engelhardt, Remarks on thermalization in 2D CFT, Phys. Rev. D 94 (2016) 126019 [arXiv: 1604.05327] [INSPIRE].

[11] A. Dymarsky and S. Sugishita, KdV-charged black holes, JHEP 05 (2020) 041 [arXiv:2002.08368] [INSPIRE].

[12] O. Fuentealba et al., Integrable systems with $B M S_{3}$ Poisson structure and the dynamics of locally flat spacetimes, JHEP 01 (2018) 148 [arXiv:1711.02646] [INSPIRE].

[13] D. Melnikov, F. Novaes, A. Pérez and R. Troncoso, Lifshitz Scaling, Microstate Counting from Number Theory and Black Hole Entropy, JHEP 06 (2019) 054 [arXiv: 1808.04034] [INSPIRE].

[14] M.P. Blencowe, A Consistent Interacting Massless Higher Spin Field Theory in D $=(2+1)$, Class. Quant. Grav. 6 (1989) 443 [INSPIRE].

[15] E. Bergshoeff, M.P. Blencowe and K.S. Stelle, Area Preserving Diffeomorphisms and Higher Spin Algebra, Commun. Math. Phys. 128 (1990) 213 [InSPIRE].

[16] M.A. Vasiliev, Higher spin gauge theories in four-dimensions, three-dimensions, and two-dimensions, Int. J. Mod. Phys. D 5 (1996) 763 [hep-th/9611024] [INSPIRE].

[17] G. Compère and W. Song, $\mathcal{W}$ symmetry and integrability of higher spin black holes, JHEP 09 (2013) 144 [arXiv: 1306.0014] [INSPIRE].

[18] M. Gutperle and Y. Li, Higher Spin Lifshitz Theory and Integrable Systems, Phys. Rev. D 91 (2015) 046012 [arXiv: 1412.7085] [INSPIRE].

[19] M. Beccaria, M. Gutperle, Y. Li and G. Macorini, Higher spin Lifshitz theories and the Korteweg-de Vries hierarchy, Phys. Rev. D 92 (2015) 085005 [arXiv: 1504.06555] [INSPIRE].

[20] J. Boussinesq, Théorie des ondes et des remous qui se propagent le long d'un canal rectangulaire horizontal, en communiquant au liquide contenu dans ce canal des vitesses sensiblement pareilles de la surface au fond, Journal de Mathématiques Pures et Appliquées (1872) 55 .

[21] V.E. Zakharov, On stochastization of one-dimensional chains of nonlinear oscillations, Sov. Phys. JETP 38 (1974) 108.

[22] D.J. Kaup, A Higher-Order Water-Wave Equation and the Method for Solving It, Prog. Theor. Phys. 54 (1975) 396.

[23] R. Hirota and J. Satsuma, Nonlinear Evolution Equations Generated from the Backlund Transformation for the Boussinesq Equation, Prog. Theor. Phys. 57 (1977) 797 [InSPIRE]. 
[24] A.P. Fordy and J. Gibbons, Factorization of Operators. 2., J. Math. Phys. 22 (1981) 1170 [INSPIRE].

[25] V.G. Drinfeld and V.V. Sokolov, Lie algebras and equations of Korteweg-de Vries type, J. Sov. Math. 30 (1984) 1975 [INSPIRE].

[26] D. Grumiller, A. Pérez, S. Prohazka, D. Tempo and R. Troncoso, Higher Spin Black Holes with Soft Hair, JHEP 10 (2016) 119 [arXiv:1607.05360] [inSPIRE].

[27] P. Mathieu and W. Oevel, The $W_{3}^{(2)}$ conformal algebra and the Boussinesq hierarchy, Mod. Phys. Lett. A 06 (1991) 2397.

[28] E.S. Fradkin and M.A. Vasiliev, On the Gravitational Interaction of Massless Higher Spin Fields, Phys. Lett. B 189 (1987) 89 [INSPIRE].

[29] M.A. Vasiliev, Consistent equation for interacting gauge fields of all spins in (3+1)-dimensions, Phys. Lett. B 243 (1990) 378 [INSPIRE].

[30] M.A. Vasiliev, Nonlinear equations for symmetric massless higher spin fields in (A)dS(d), Phys. Lett. B 567 (2003) 139 [hep-th/0304049] [INSPIRE].

[31] M. Henneaux and S.-J. Rey, Nonlinear $W_{\infty}$ as Asymptotic Symmetry of Three-Dimensional Higher Spin Anti-de Sitter Gravity, JHEP 12 (2010) 007 [arXiv:1008.4579] [INSPIRE].

[32] A. Campoleoni, S. Fredenhagen, S. Pfenninger and S. Theisen, Asymptotic symmetries of three-dimensional gravity coupled to higher-spin fields, JHEP 11 (2010) 007 [arXiv: 1008.4744] [INSPIRE].

[33] O. Coussaert, M. Henneaux and P. van Driel, The asymptotic dynamics of three-dimensional Einstein gravity with a negative cosmological constant, Class. Quant. Grav. 12 (1995) 2961 [gr-qc/9506019] [INSPIRE].

[34] T. Regge and C. Teitelboim, Role of Surface Integrals in the Hamiltonian Formulation of General Relativity, Annals Phys. 88 (1974) 286 [INSPIRE].

[35] M. Henneaux, A. Pérez, D. Tempo and R. Troncoso, Chemical potentials in three-dimensional higher spin anti-de Sitter gravity, JHEP 12 (2013) 048 [arXiv: 1309.4362] [INSPIRE].

[36] C. Bunster, M. Henneaux, A. Pérez, D. Tempo and R. Troncoso, Generalized Black Holes in Three-dimensional Spacetime, JHEP 05 (2014) 031 [arXiv:1404.3305] [INSPIRE].

[37] M. Gutperle and P. Kraus, Higher Spin Black Holes, JHEP 05 (2011) 022 [arXiv:1103.4304] [INSPIRE].

[38] M. Ammon, M. Gutperle, P. Kraus and E. Perlmutter, Spacetime Geometry in Higher Spin Gravity, JHEP 10 (2011) 053 [arXiv:1106.4788] [INSPIRE].

[39] M. Henneaux, A. Pérez, D. Tempo and R. Troncoso, Hypersymmetry bounds and three-dimensional higher-spin black holes, JHEP 08 (2015) 021 [arXiv:1506.01847] [INSPIRE].

[40] M. Bañados, A. Castro, A. Faraggi and J.I. Jottar, Extremal Higher Spin Black Holes, JHEP 04 (2016) 077 [arXiv: 1512.00073] [INSPIRE].

[41] M. Henneaux, L. Maoz and A. Schwimmer, Asymptotic dynamics and asymptotic symmetries of three-dimensional extended AdS supergravity, Annals Phys. 282 (2000) 31 [hep-th/9910013] [INSPIRE]. 
[42] P. Forgacs, A. Wipf, J. Balog, L. Feher and L. O'Raifeartaigh, Liouville and Toda Theories as Conformally Reduced WZNW Theories, Phys. Lett. B 227 (1989) 214 [inSPIRE].

[43] A. Alekseev and S.L. Shatashvili, Path Integral Quantization of the Coadjoint Orbits of the Virasoro Group and 2D Gravity, Nucl. Phys. B 323 (1989) 719 [InSPIRE].

[44] E. Witten, Quantum Field Theory and the Jones Polynomial, Commun. Math. Phys. 121 (1989) 351 [INSPIRE].

[45] S. Elitzur, G.W. Moore, A. Schwimmer and N. Seiberg, Remarks on the Canonical Quantization of the Chern-Simons-Witten Theory, Nucl. Phys. B 326 (1989) 108 [INSPIRE].

[46] H.A. González, J. Matulich, M. Pino and R. Troncoso, Revisiting the asymptotic dynamics of General Relativity on $A d S_{3}$, JHEP 12 (2018) 115 [arXiv: 1809.02749] [InSPIRE].

[47] D. Grumiller and W. Merbis, Near horizon dynamics of three dimensional black holes, SciPost Phys. 8 (2020) 010 [arXiv: 1906. 10694] [INSPIRE].

[48] R. Floreanini and R. Jackiw, Selfdual Fields as Charge Density Solitons, Phys. Rev. Lett. 59 (1987) 1873 [INSPIRE].

[49] R. Sasaki and I. Yamanaka, Virasoro Algebra, Vertex Operators, Quantum Sine-Gordon and Solvable Quantum Field Theories, Adv. Stud. Pure Math. 16 (1988) 271.

[50] T. Eguchi and S.-K. Yang, Deformations of Conformal Field Theories and Soliton Equations, Phys. Lett. B 224 (1989) 373 [inSPIRE].

[51] V.V. Bazhanov, S.L. Lukyanov and A.B. Zamolodchikov, Integrable structure of conformal field theory, quantum KdV theory and thermodynamic Bethe ansatz, Commun. Math. Phys. 177 (1996) 381 [hep-th/9412229] [INSPIRE].

[52] P. Calabrese, F.H.L. Essler and M. Fagotti, Quantum Quench in the Transverse Field Ising Chain, Phys. Rev. Lett. 106 (2011) 227203 [arXiv:1104.0154] [InSPIRE].

[53] S. Sotiriadis and P. Calabrese, Validity of the GGE for quantum quenches from interacting to noninteracting models, J. Stat. Mech. 1407 (2014) P07024 [arXiv:1403.7431] [InSPIRE].

[54] E. Ilievski, J. De Nardis, B. Wouters, J.-S. Caux, F.H.L. Essler and T. Prosen, Complete generalized gibbs ensembles in an interacting theory, Phys. Rev. Lett. 115 (2015) 157201.

[55] L. Vidmar and M. Rigol, Generalized gibbs ensemble in integrable lattice models, J. Stat. Mech. 2016 (2016) 064007.

[56] B. Pozsgay, E. Vernier and M.A. Werner, On generalized gibbs ensembles with an infinite set of conserved charges, J. Stat. Mech. 2017 (2017) 093103.

[57] A. Dymarsky and K. Pavlenko, Generalized Gibbs Ensemble of 2d CFTs at large central charge in the thermodynamic limit, JHEP 01 (2019) 098 [arXiv:1810.11025] [INSPIRE].

[58] A. Maloney, G.S. Ng, S.F. Ross and I. Tsiares, Thermal Correlation Functions of KdV Charges in 2D CFT, JHEP 02 (2019) 044 [arXiv: 1810.11053] [INSPIRE].

[59] A. Maloney, G.S. Ng, S.F. Ross and I. Tsiares, Generalized Gibbs Ensemble and the Statistics of KdV Charges in 2D CFT, JHEP 03 (2019) 075 [arXiv: 1810.11054] [InSPIRE].

[60] A. Dymarsky and K. Pavlenko, Exact generalized partition function of 2D CFTs at large central charge, JHEP 19 (2020) 077 [arXiv: 1812.05108] [INSPIRE].

[61] E.M. Brehm and D. Das, Korteweg-de Vries characters in large central charge CFTs, Phys. Rev. D 101 (2020) 086025 [arXiv:1901.10354] [INSPIRE]. 
[62] A. Dymarsky and K. Pavlenko, Generalized Eigenstate Thermalization Hypothesis in 2D Conformal Field Theories, Phys. Rev. Lett. 123 (2019) 111602 [arXiv:1903.03559] [INSPIRE].

[63] A. Pérez, D. Tempo and R. Troncoso, work in progress.

[64] L.A. Dickey, Soliton Equations And Hamiltonian Systems, Advanced Series in Mathematical Physics, World Scientific, (2003), [DOI].

[65] A. Maloney and E. Witten, Averaging over Narain moduli space, JHEP 10 (2020) 187 [arXiv: 2006. 04855] [INSPIRE].

[66] N. Afkhami-Jeddi, H. Cohn, T. Hartman and A. Tajdini, Free partition functions and an averaged holographic duality, arXiv:2006.04839 [INSPIRE].

[67] J. Cotler and K. Jensen, $A d S_{3}$ gravity and random $C F T$, arXiv:2006.08648 [INSPIRE].

[68] S. Bhattacharyya, V.E. Hubeny, S. Minwalla and M. Rangamani, Nonlinear Fluid Dynamics from Gravity, JHEP 02 (2008) 045 [arXiv:0712.2456] [INSPIRE].

[69] M. Haack and A. Yarom, Nonlinear viscous hydrodynamics in various dimensions using AdS/CFT, JHEP 10 (2008) 063 [arXiv:0806.4602] [INSPIRE].

[70] S. Bhattacharyya, R. Loganayagam, I. Mandal, S. Minwalla and A. Sharma, Conformal Nonlinear Fluid Dynamics from Gravity in Arbitrary Dimensions, JHEP 12 (2008) 116 [arXiv: 0809.4272] [INSPIRE].

[71] V.E. Hubeny, S. Minwalla and M. Rangamani, The fluid/gravity correspondence, in Theoretical Advanced Study Institute in Elementary Particle Physics: String theory and its Applications: From meV to the Planck Scale, pp. 348-383, 2012 [arXiv:1107.5780] [INSPIRE].

[72] A. Campoleoni, L. Ciambelli, C. Marteau, P.M. Petropoulos and K. Siampos, Two-dimensional fluids and their holographic duals, Nucl. Phys. B 946 (2019) 114692 [arXiv: 1812.04019] [INSPIRE].

[73] M. Henneaux, W. Merbis and A. Ranjbar, Asymptotic dynamics of $A d S_{3}$ gravity with two asymptotic regions, JHEP 03 (2020) 064 [arXiv: 1912.09465] [INSPIRE].

[74] B.B. Kadomtsev and V.I. Petviashvili, On the Stability of Solitary Waves in Weakly Dispersing Media, Sov. Phys. Dokl. 15 (1970) 539.

[75] H. Afshar, A. Bagchi, R. Fareghbal, D. Grumiller and J. Rosseel, Spin-3 Gravity in Three-Dimensional Flat Space, Phys. Rev. Lett. 111 (2013) 121603 [arXiv:1307.4768] [INSPIRE].

[76] H.A. González, J. Matulich, M. Pino and R. Troncoso, Asymptotically flat spacetimes in three-dimensional higher spin gravity, JHEP 09 (2013) 016 [arXiv:1307.5651] [INSPIRE].

[77] M. Gary, D. Grumiller, M. Riegler and J. Rosseel, Flat space (higher spin) gravity with chemical potentials, JHEP 01 (2015) 152 [arXiv:1411.3728] [INSPIRE].

[78] J. Matulich, A. Pérez, D. Tempo and R. Troncoso, Higher spin extension of cosmological spacetimes in 3D: asymptotically flat behaviour with chemical potentials and thermodynamics, JHEP 05 (2015) 025 [arXiv: 1412.1464] [INSPIRE].

[79] M. Ammon, D. Grumiller, S. Prohazka, M. Riegler and R. Wutte, Higher-Spin Flat Space Cosmologies with Soft Hair, JHEP 05 (2017) 031 [arXiv: 1703.02594] [InSPIRE]. 
[80] O. Fuentealba, J. Matulich and R. Troncoso, Extension of the Poincaré group with half-integer spin generators: hypergravity and beyond, JHEP 09 (2015) 003 [arXiv: 1505.06173] [INSPIRE].

[81] O. Fuentealba, J. Matulich and R. Troncoso, Asymptotically flat structure of hypergravity in three spacetime dimensions, JHEP 10 (2015) 009 [arXiv: 1508.04663] [INSPIRE].

[82] M. Henneaux, A. Pérez, D. Tempo and R. Troncoso, Extended anti-de Sitter Hypergravity in $2+1$ Dimensions and Hypersymmetry Bounds, in International Workshop on Higher Spin Gauge Theories, pp. 139-157, 2017, DOI [arXiv:1512.08603] [INSPIRE].

[83] M. Antonowicz, A.P. Fordy and Q.P. Liu, Energy-dependent third-order lax operators, Nonlinearity 4 (1991) 669. 